\title{
Unstructured Cartesian refinement with sharp interface immersed boundary method for 3D unsteady incompressible flows
}

\author{
Dionysios Angelidis $^{1}$, Saurabh Chawdhary ${ }^{1,2}$ and Fotis Sotiropoulos ${ }^{1,3, *}$ \\ ${ }^{1}$ St. Anthony Falls Laboratory, College of Science and Engineering, University of Minnesota, Minneapolis, Minnesota 55414, USA \\ ${ }^{2}$ Department of Mechanical Engineering, University of Minnesota, Minneapolis, Minnesota 55455, USA \\ ${ }^{3}$ Department of Civil Engineering, College of Engineering and Applied Sciences, Stony Brook University, Stony Brook, New York
} 11794, USA

\begin{abstract}
A novel numerical method is developed for solving the 3D, unsteady, incompressible Navier-Stokes equations on locally refined fully unstructured Cartesian grids in domains with arbitrarily complex immersed boundaries. Owing to the utilization of the fractional step method on an unstructured Cartesian hybrid staggered/non-staggered grid layout, flux mismatch and pressure discontinuity issues are avoided and the divergence free constraint is inherently satisfied to machine zero. Auxiliary/hanging nodes are used to facilitate the discretization of the governing equations. The second-order accuracy of the solver is ensured by using multi-dimension Lagrange interpolation operators and appropriate differencing schemes at the interface of regions with different levels of refinement. The sharp interface immersed boundary method is augmented with local near-boundary refinement to handle arbitrarily complex boundaries. The discrete momentum equation is solved with the matrix free Newton-Krylov method and the Krylov-subspace method is employed to solve the Poisson equation. The second-order accuracy of the proposed method on unstructured Cartesian grids is demonstrated by solving the Poisson equation with a known analytical solution. A number of three-dimensional laminar flow simulations of increasing complexity illustrate the ability of the method to handle flows across a range of Reynolds numbers and flow regimes. Laminar steady and unsteady flows past a sphere and the oblique vortex shedding from a circular cylinder mounted between two end walls demonstrate the accuracy, the efficiency and the smooth transition of scales and coherent structures across refinement levels. Large-eddy simulation (LES) past a miniature wind turbine rotor, parameterized using the actuator line approach, indicates the ability of the fully unstructured solver to simulate complex turbulent flows. Finally, a geometry resolving LES of turbulent flow past a complete hydrokinetic turbine illustrates the potential of the method to simulate turbulent flows past geometrically complex bodies on locally refined meshes. In all the cases, the results are found to be in very good agreement with published data and savings in computational resources are achieved.
\end{abstract}

(C) 2016 Published by Elsevier Ltd.

Keywords: Unstructured Cartesian grids, Adaptive mesh refinement, Immersed boundaries, Finite-difference method, Incompressible flows

\section{Introduction}

Real-life flow problems in engineering and biological applications involve stationary or moving arbitrarily complex geometries and occur across a range of Reynolds numbers and flow regimes. Typical examples include the hydrodynamics of aquatic thrust generation around cephalopod swimming [34], the hemodynamics in arbitrarily complex cardiovascular anatomies [22] and turbulent flows over wind farms in complex terrains [77]. Conforming grids can be used to simulate flows around stationary and morphologically simple bodies. However, body-fitted mesh generation around moving complex geometries constitutes a non-trivial task which often results in poor quality grids that downgrade the robustness of the solver and the accuracy of the calculations. On the other hand, immersed boundary methods have emerged as a powerful tool for handling arbitrarily complex flows in various engineering and biological applications [61]. Nevertheless, a major limitation of such methods, is their inherent inability to cluster grid nodes in the vicinity of solid boundaries, which is required to accurately resolve the near-wall viscosity dominated region at Reynolds numbers of practical interest. Integrating immersed boundary methods with local, manual or adaptive, mesh refinement provides a powerful approach for mitigating this difficulty. Such undertaking, however, requires developing solvers capable of accurately solving the 3D unsteady incompressible Navier-Stokes equations on locally refined meshes. In this paper we develop such a method and integrate it with a sharp interface immersed boundary method to propose a versatile approach for handling problems with arbitrarily complex boundaries.

*Corresponding author: fotis.sotiropoulos@stonybrook.edu 
Numerous methods have been proposed handling complex geometries using adaptive spatial discretization techniques. Such methods are typically classified according to the topological connection of the sub-domains, as: (i) overlapping decomposition; (ii) non-overlapping decomposition and (iii) non-decomposed methods. The efficiency of each of them is determined by the algorithmic simplicity, the capability of smooth transition across grid resolution interfaces and the parallelization potential. In the overlapping decomposition methods, which include hierarchical adaptive mesh refinement (AMR) techniques [10, 14, 67] and overset grids [63, 8, 64, 66, 79], the sub-domains have interior points in common. On the other hand, multi-block [46, 49, 20, 4] and patched grid techniques [52, 28, 9] are examples of non-overlapping decomposition or substructuring methods. An alternative to the domain decomposition techniques (DDM) is the implementation of monolithic structured or unstructured grids [18, 7].

Hierarchical AMR methods manage overlapping grids and take advantage of the tree-based topology of aligned overlaid patches. The pioneering adaptive mesh refinement approach for solving 1D and 2D hyperbolic partial differential equations (PDEs), was presented almost three decades ago in [11]. In this work, tree-based hierarchical data structures are utilized to adaptively increase the numerical accuracy of the calculations, where an error estimate is large. Berger and Colella [10] developed a 2D AMR strategy for solving time dependent shock hydrodynamics problems. The first attempt to solve laminar and turbulent flows, using the Reynolds-averaged incompressible Navier-Stokes equations, on locally structured overlaid grids was reported by Caruso et al. [14]. Chen et al. [17] implemented an AMR algorithm for solving both laminar and turbulent flows in two dimensions and investigated its efficiency as a function of the AMR sensor. In the work of Theodorakakos and Bergeles [67], an AMR generalized curvilinear technique enabled the performance of large scale calculations around complex topographies. Several scientific packages have been developed to manage dynamic data structures and simplify the solution of the governing equations, under the AMR framework [51, 74, 10, 37]. Vanella et al. [69], for instance, used the grid topology and data-structures established by the Paramesh toolkit [37] to perform fluid-structure interaction simulations for laminar and turbulent incompressible flows. Major limitations of the hierarchical AMR methods is the increased algorithmic complexity attributed to the required restriction/prolongation operations and the non-inherent satisfaction of the conservation properties.

To alleviate the constraints of managing aligned overlapped locally structured grids, overset or Chimera grids allow grids to be overlapped without any restriction on the relative position and orientation of the external borders of blocks. The use of overset grids was initially proposed by Starius [62, 63] who developed composite mesh difference methods (CMDM) to solve elliptic and hyperbolic differential equations for the inviscid shallow-water equations. Benek et al. and Steger [8, 64] adopted the Chimera approach for high Reynolds calculations over complex geometries. Meakin and Suhs [41] presented a method for the simulation of three-dimensional unsteady viscous flows with relative motion of bodies and grid systems. Connection between different grids is often achieved by interpolating the flow variables between the overlapped domains [8, 29]; however this approach implies that conservation is not strictly enforced. Implicit calculations involve an iterative procedure to couple the solution but such algorithms suffer from slow convergence rate and the individual systems can be singular [13]. Several mass-flux based interpolation (MFBI) algorithms have been proposed to ensure consistency of the mass at the interface between Chimera grids. Some algorithms use approximate mass conservation formulas [66], other require correction of the interpolated velocity field to ensure global mass conservation [79] and other schemes are based on the idea of local mesh reconstruction to facilitate the discretization of the equations between multiblock interface boundaries [23]. While overset grid methods constitute a powerful approach to handle complex geometries, the inherent algorithmic complexity coupled with the non-strict conservation make Chimera grids still not competitive with unstructured grids.

In the multi-block strategy, where the computational domain is decomposed, into several blocks, individual domains are not allowed to overlap and grid lines join across adjacent blocks. This method can be considered an alternative to hierarchical AMR or unstructured methods as it can result to same spatial resolution. De Nicola et al. studied 1D [21] and 2D [46] advection-diffusion problems and showed that the block interface could create instability in the numerical solution. Pascarelli et al. [49] investigated the feasibility of the multi-block approach to solve time-developing turbulent boundary layers over an isothermal flat plate. Their study revealed that multi-block calculations are computationally less demanding compared to single-block calculations performed on structured grids. However, they adopted an explicit time advancement scheme since implicit discretization would require iterative solution of the governing equations. Darbandi and Naderi [20] proposed a hybrid multi-block finite volume method and demonstrated that their methodology is flexible in mesh generation and can satisfy the conservation properties through the block boundaries on laminar flows. In the more recent work of Anupindi et al. [4] a multi-block approach was combined with an immersed boundary method to perform LES of arterial hemodynamics. In this study, only equal blocks with uniform meshes were utilized to avoid any interpolation at the block-interfaces and make the scheme conservative.

The patched grid approach (PG) is a non-overlapping domain decomposition strategy according to which individual grids are not overlapped and non-coincident interfaces (zonal boundaries) are resolved. It was Rai [52] who initially demonstrated that the PG scheme can be conservative. Hessenius [28] extended the zonal scheme [52] in two dimensions to solve the flow around a double-airfoil configuration using individual patched grid systems. Benkenida et al. [9] evaluated the performance of the AMR and PG strategies by using comparable number of grid points. Numerical calculations of the flow around an aircraft model revealed that even though AMR calculations were faster, the vortex characteristics could not be sufficiently captured in the AMR computations, due to the local characteristics of the AMR technique.

Unstructured meshes allow flexible spatial adaptivity [18,7]; however generating good quality high resolution meshes 


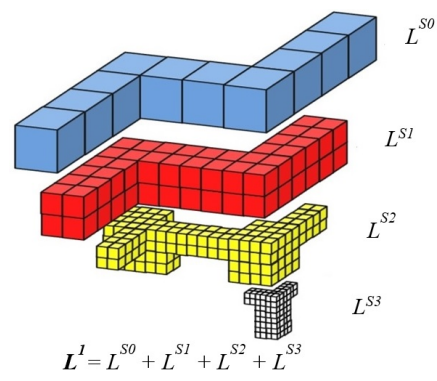

(a)

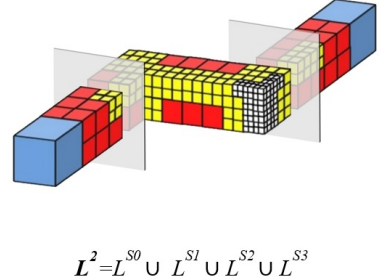

(b)

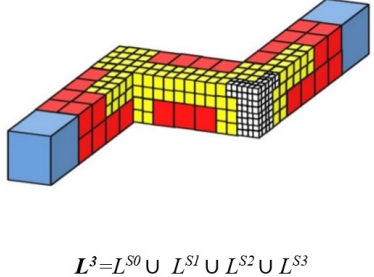

(c)

Fig. 1: Spatial adaptively refined grids using: (a) Overlapped domain decomposition (AMR); (b) multi-block domain decomposition and (c) fully unstructured arrangement.

close to complex boundaries is far from trivial. Monolithic and fully unstructured treatment of adaptively refined Cartesian grids benefit from simple grid generation and facilitate the global conservation. Coelho et al. [19] reported a method for solving the 2D incompressible Navier-Stokes equations with strong coupling between cells of varying refinement levels, by discretizing the flow equations using a finite volume method, on fully unstructured collocated grids. Papadakis and Bergeles [48] extended the work of [19] and introduced a low-order fully unstructured method for solving 3D turbulent incompressible flows, covering the computational domain by block-structured subgrids. Inspired from the previous works, Anagnostopoulos [1] discretized the transport equations on arbitrary refined 2D Cartesian grids, satisfying, at the same time, the divergence free constraint. Recently, Angelidis et al. [2] presented a 2D fully unstructured Cartesian incompressible flow solver to investigate laminar canonical flows and the turbulent air mixing potential in idealized street canyons for several thermal stratification scenarios [45].

As is evident from above discussion, incompressible Navier-Stokes solvers which handle adaptive spatial discretization suffer from the challenging issues of pressure discontinuity and ensuring global mass conservation. The overlapping decomposition methods require restriction/prolongation operations to enforce the divergence-free constraint whereas the non-overlapping decomposition methods cannot strictly ensure global mass conservation. In this paper we develop a novel numerical method for discretizing and solving the 3D, unsteady, incompressible Navier-Stokes equations in fully unstructured Cartesian grids resulting when local mesh refinement is implemented in domains with arbitrarily complex immersed boundaries. We generalize the hybrid staggered/non-staggered grid layout originally proposed by [26] for structured Cartesian grids to unstructured Cartesian grids and develop a framework for constructing finite-difference formulas across interface regions with different levels of refinement. Our approach employs auxiliary/hanging nodes and multi-dimensional Lagrange interpolation operators. While the method we develop herein is designed to be second-order accurate in space, the proposed discretization framework is general and can be used to develop discrete approximations of any desired order of accuracy on unstructured Cartesian grids by adjusting the size of computational stencils and interpolation formulas. The sharp interface immersed boundary method of Gilmanov and Sotiropoulos [26] and Borazjani et al. [12] is extended to be applicable to unstructured Cartesian grids with local near-boundary mesh refinement resulting in a more accurate and efficient methodology for resolving near-wall flows past arbitrarily complex boundaries. This is accomplished by developing an approach for reconstructing boundary conditions for the pressure and velocity fields at grid nodes in the immediate vicinity of immersed boundaries using the concept of hanging nodes. The resulting discrete governing equations on unstructured Cartesian grids are integrated in time using a monolithic fractional step method. Therefore, challenges that plague other local refinement methodologies [69], such as flux mismatch and pressure discontinuity across interfaces of varying resolution, are inherently eliminated and the divergence free constraint is driven to machine zero. To maintain algorithmic simplicity, grid refinement is limited to isotropic octree division albeit recursively. We exploit the Rubik's cube analogy to define the grid topology while octree hierarchical structures could also be used.

Incorporating the hybrid staggered/non-staggered layout with a monolithic fully unstructured arrangement has the following benefits: a) the hybrid approach retains all the desirable features of the staggered grid arrangement but also simplifies the discretization of the governing equations, b) the implementation of the sharp interface immersed boundary method [26, 25, 12] is facilitated and c) partitioning of the computational domain can be performed in a straightforward manner, by providing the partitioner with the adjacency list. Parallelism is achieved using the PETSc libraries [5, 6]. The accuracy of the calculations is increased by refining the mesh in the vicinity of stationary or moving immersed boundaries with low computational cost. Overall, the proposed unstructured solver is designed to enable high fidelity and low cost direct numerical simulations (DNS) and large-eddy simulations (LES) of 3D unsteady incompressible flows past geometrically complex bodies on locally refined meshes.

The paper is organized as follows. In Section 2 we discuss the numerical method and describe the grid topology and the discretization procedure on the unstructured mesh. Section 3 is dedicated on the implementation of sharp interface immersed boundary method on adaptively refined grids. In Section 4 we report evidence to demonstrate the efficiency and accuracy of our method. In particular, in Section 4.1 the second-order accuracy and robustness of the Poisson solver on unstructured meshes are investigated by solving a problem for which analytical solution of the Poisson equation exists. In Section 4.2 the flow around a stationary sphere, within a range of $20 \leq R e \leq 300$, is studied and we demonstrate the 
accuracy and efficiency of the flow solver in simulating flows with complex vortex shedding. In Section 4.3 the three-dimensional flow past a circular cylinder mounted between two end walls is simulated with emphasis on the ability of the solver to accurately capture the rich shedding dynamics in the wake of the cylinder and on the smooth transition of coherent structures of varying temporal and spatial scales across multiple interfaces of grid resolution. In Section 4.4 we perform LES to simulate the turbulent flow field past a miniature turbine. We demonstrate that our method can capture the rich dynamics in the wake of the turbine, with actuator models and low computational cost, and our results agree well with published experimental and numerical results. In Section 4.5 we illustrate the ability of the method to carry out LES on locally refined grids past a complete hydrokinetic turbine resolving all the geometrical details of the turbine with the sharp interface immersed boundary method of [26, 25, 12]. Finally, in Section 5, we summarize the major findings of our work and discuss the ongoing studies which focus on extending the capabilities of the presented method.

\section{The numerical method}

\subsection{The governing equations}

We solve the unsteady incompressible Navier-Stokes equations on Cartesian unstructured grids. The governing equations are presented here, for the sake of generality, in their spatially-filtered form for LES in compact tensor form (repeated indices imply summation) as follows:

$$
\begin{gathered}
\frac{\partial \bar{u}_{i}}{\partial x_{i}}=0 \\
\frac{\partial \bar{u}_{i}}{\partial t}+\frac{\partial \bar{u}_{i} \bar{u}_{j}}{\partial x_{i}}=-\frac{\partial \bar{p}}{\partial x_{i}}+\frac{1}{\operatorname{Re}} \frac{\partial^{2} \bar{u}_{i}}{\partial x_{j} \partial x_{j}}-\frac{\partial \tau_{i j}}{\partial x_{j}}+f_{i}
\end{gathered}
$$

where $\bar{u}_{i}(i=1,2,3)$ denote the resolved velocity components in $x_{i}$ Cartesian coordinates, $\bar{p}$ is the resolved pressure divided by the density $\rho$ and $R e$ is the Reynolds number based on the characteristic length and velocity scales. The sub-grid scales (SGS) are modeled by utilizing the Smagorinsky-Lilly model [58] while the calculation of the subgrid tensor $\tau_{i j}$ is based on the assumption that the filter width is equal to the grid spacing and the Smagorinsky constant is $C_{s}=0.1$ (for direct numerical simulations this term is zero). The term $f_{i}$ represents the contribution of external forces to the flow field; for instance the effect of the rotation of the wind turbine blades can be introduced by calculating the distributed forces according to an actuator line model (AL) [77].

\subsection{Grid topology}

Grid refinement strategies can be classified according to the topological connection of the refined regions which determine the efficiency and robustness of the solver. In Fig. 1 overlapped, non-overlapped and fully unstructured grid topologies are shown, for refined Cartesian grids. Assuming that $L^{S i}, i=0,1 . ., i_{\text {max }}$ are the individual grid blocks at different refinement levels, the information to be managed during the solution on overlapped decomposed grids is $L^{1}=L^{S 0}+L^{S 1}+\ldots+L^{S i_{\max }}$. On the contrary, when multi-block or unstructured grids are used, the memory requirements are kept low since non- overlapping $L^{2}=L^{3}=L^{S 0} \cup L^{S 1} \cup \ldots \cup L^{S i_{\max }}$ information needed for solving the governing equations.

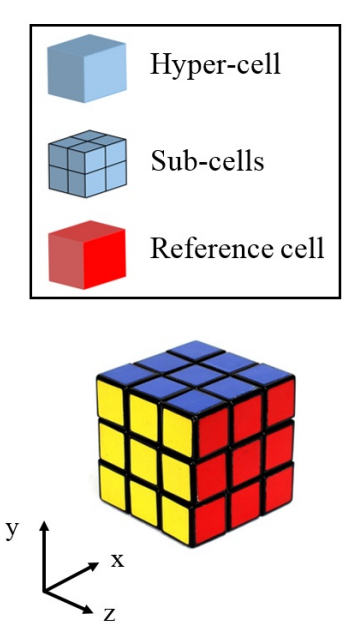

(a)

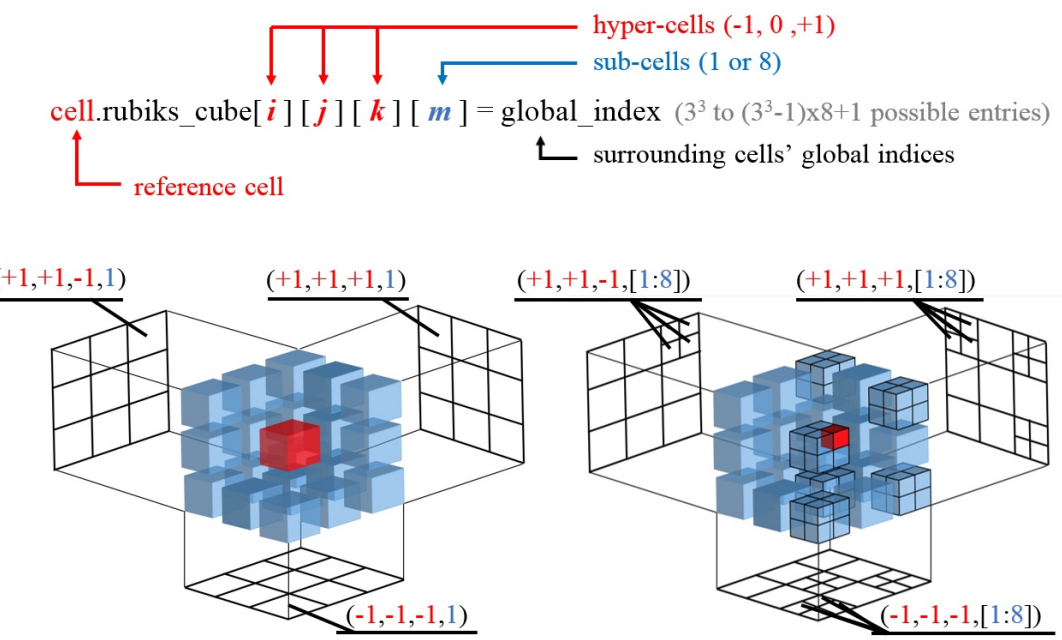

(b) (c)

Fig. 2: The Rubik's cube analogy employed to describe the grid's topology around a reference cell: (a) Rubik's cube; (b) grid's topology without refinement and (c) grid's topology with refinement. 
To describe the way the grid connectivity is established, we may assume that a reference cell is located in the middle of a Rubik's cube (Fig. 2a,b). Hence, we can locally define the mesh topology on locally structured grids. If this idea is extended to multi-resolution grids, all the 27 cells of the Rubik's cube can be considered as hyper-cells and they can be further refined. By doing so, a locally structured $3 \times 3 \times 3$ mesh may describe the position of any hyper-cell and we can readily access any refined component of a hyper-cell that may be called sub-cell. In order to achieve smooth transition between the levels of refinement and maintain algorithmic simplicity, the allowed difference between the refinement level of the hyper-cells cannot be larger than one. The structure cell in our method contains any information needed to define local or global indices regarding the local grid topology. In this way, any local or global indices can be easily obtained by using a structure like cell.rubiks_cube $[i][j][k][m]$ where $i, j, k=\{-1,0,1\}$ indicate the position of a hyper-cell and $m \in[1,8]$ the corresponding sub-cells, if any (Fig. 2k). It should be noted that the total number of cells included in the $3 \times 3 \times 3$ hyper-cells is the maximum information needed to generate the support stencil needed for all the calculations. Several other approaches, including the oct-tree hierarchical methods, could be used to define the multi-resolution grid topology. However, our goal here is to avoid storing any overlapped information and keep the memory cost low.

To manage the unstructured staggered arrangement, global indices are defined at the cell centers as well as the face centers. Whenever neighboring cells have different levels of refinement, face indices are defined for the four smaller faces. The adopted monolithic single-block arrangement enables straightforward partition of the computational domain, which is crucial to achieve load balance and control the scalability potential of the solver. All the variables of the staggered arrangement are stored in one-dimensional vectors and the parallelization of the calculations is achieved by distributing the vectors in accordance to the domain decomposition. The adjacency list of the unstructured grid is modified every time the grid topology is changing and is provided to the partitioner for domain decomposition.

\subsection{Lagrange reconstruction around cells of varying resolution}

To facilitate the discretization procedure in the vicinity of cells surrounded by others of different resolution and calculate the flow variables at hanging nodes, we employ herein the Lagrange interpolation formula, as it ensures that the interpolated function goes through the given data set and the accuracy of the calculations is determined by the size of the computational stencil. Provided that the Lagrange basis polynomials can be described as:

$$
L_{i}(\omega)=\prod_{s=0, s \neq 1}^{n_{\omega}} \frac{\omega-\omega_{s}}{\omega_{i}-\omega_{s}}, 0 \leq i \leq n_{\omega}
$$

where $\omega$ can be any Cartesian coordinate $\xi, \eta$ and $\left(n_{\omega}+1\right)$ is the number of data points, the 1D interpolation can be defined as:

$$
\phi(\xi)=\sum_{i=0}^{n_{\xi}} f\left(\xi_{i}\right) L_{i}(\xi)
$$

the $2 \mathrm{D}$ interpolation will be:

$$
\phi(\xi, \eta)=\sum_{i=0}^{n_{\xi}} \sum_{j=0}^{n_{\eta}} f\left(\xi_{i}, \eta_{j}\right) L_{i}(\xi) L_{j}(\eta)
$$

The above Lagrange interpolant polynomials interpolate $\left(n_{\xi}+1\right)$ or $\left(n_{\xi}+1\right) \times\left(n_{\eta}+1\right)$ data points $f\left(\xi_{i}\right)$ or $f\left(\xi_{i}, \eta_{j}\right)$ respectively. Essentially, the interpolated values derived from the Lagrangian basis polynomials constitute a correlation between weighting factors and the information from the data points.

We perform second order accurate interpolations by utilizing the 1D Lagrange formula on a three-point stencil which results in a quadratic reconstruction and perform 2 D Lagrange interpolation on a $3 \times 3$ stencil resulting in a bi-quadratic quadrilateral reconstruction. Thus, the equivalent polynomial reconstruction of the Lagrangian interpolations enables the definition of weighting factors when points of the reconstruction stencil lie along the boundaries of the domain governed either by Dirichlet or Neumann conditions. For this reason, the 2D Lagrange interpolation may also be expressed as:

$$
\phi(\xi, \eta)=\sum_{i=0}^{n_{\xi}} \sum_{j=0}^{n_{\eta}} f\left(\xi_{i}, \eta_{j}\right) w_{i j}(\xi, \eta)
$$

and the 2D Lagrange operator can be defined as:

$$
\mathcal{S}(.)=\sum_{i=0}^{n_{\xi}} \sum_{j=0}^{n_{\eta}}(.)_{\xi_{i}, \eta_{j}} w_{i j}(\xi, \eta)
$$

Around any reference cell, $3 \times 3$ support stencils can be formed from the cell centered values of the surrounding hyper-cells, which are coplanar and parallel to its six faces (Fig. 2). However, if the hyper-cells are split then the hyper-cells' centered values are obtained by performing averaging from the cell centered values of the corresponding isotropically refined cells, 
which essentially represents trilinear interpolation on uniform stencils. Here it should be noted that the weighting factors $w_{i j}(\xi, \eta)$ have constant values on any isotropically refined mesh for a specific relative position and their calculation is presented in Appendix A. Hence, no additional cost is added to the flow solver for calculating the contribution of the surrounding to the hanging nodes. Our method is general and different interpolation functions or stencil sizes may be used. This provides additional flexibility to achieve the desired level of efficiency and accuracy.

\subsection{Second order differencing operator}

We discuss herein the approach we employ to construct finite difference approximations. We note that when all the neighboring cells have the same level of refinement, three-point central finite difference formulas can be readily used. Near cells with varying grid resolution, however, a different approach needs to be employed. Let us assume that a dependent variable $\phi(\xi)$, may be represented by a function $f_{i}=f\left(\xi_{i}\right)=(.)_{i}$ which is continuous and $n$ times differentiable in an interval $\xi \in\left[-h_{i-2}, h_{i+1}\right]$; the Taylor expansion around the point $i$ for $f_{i+1}, f_{i-1}, f_{i-2}$ would give:

$$
\begin{gathered}
f_{i+1}=f_{i}+h_{i+1} f_{i}^{\prime}+\frac{h_{i+1}^{2}}{2} f_{i}^{\prime \prime}+\frac{h_{i+1}^{3}}{6} f_{i}^{\prime \prime \prime}+\frac{h_{i+1}^{4}}{24} f_{i}^{I V}+\ldots+\frac{h_{i+1}^{n}}{n !} f_{i}^{n} \\
f_{i-1}=f_{i}-h_{i-1} f_{i}^{\prime}+\frac{h_{i-1}^{2}}{2} f_{i}^{\prime \prime}-\frac{h_{i-1}^{3}}{6} f_{i}^{\prime \prime \prime}+\frac{h_{i-1}^{4}}{24} f_{i}^{I V}+\ldots+\frac{h_{i}^{n}}{n !} f_{i}^{n} \\
f_{i-2}=f_{i}-h_{i-2} f_{i}^{\prime}+\frac{h_{i-2}^{2}}{2} f_{i}^{\prime \prime}-\frac{h_{i-2}^{3}}{6} f_{i}^{\prime \prime \prime}+\frac{h_{i-2}^{4}}{24} f_{i}^{I V}+\ldots+\frac{h_{i}^{n}}{n !} f_{i}^{n}
\end{gathered}
$$

From $88-(10)$, if we neglect the contribution of the terms $f^{\prime \prime \prime}$ and higher, $f_{i}^{\prime}=\delta_{\xi}(.)_{i}$ can be evaluated from a three-point stencil having non uniform intervals with $2^{\text {nd }}$ order accuracy. The differencing operator $\delta_{\xi}$ will now be of the following general form:

$$
\delta_{\xi}(.)_{i}=\frac{c_{0} \mathcal{S}(.)_{i+1}+c_{1} \mathcal{S}(.)_{i-1}+c_{2} \mathcal{S}(.)_{i-2}}{\Delta \xi}+O\left(\Delta \xi^{2}\right)
$$

with $c_{0}, c_{1}, c_{2}=g\left(h_{i+1}, h_{i-1}, h_{i-2}\right)$. The latter equation enables the calculation of $f^{\prime}$ with $2^{\text {nd }}$ order accuracy in the middle of faces adjacent to cells with varying grid resolution. This is feasible by using 1D non-uniform stencils and ghost nodes, which will be explained in a following paragraph.

\subsection{The unstructured hybrid staggered/non-staggered grid layout}

We develop a 3D unstructured hybrid staggered/non-staggered grid layout to facilitate the satisfaction of the discrete divergence free constraint and simplify the imposition of boundary conditions in the context of the immersed boundary method. The idea of the hybrid staggered/non-staggered approach was initially proposed by [26, 25] but was restricted to structured Cartesian grids. However, the discretization of the governing equations and the employment of the fractional step method on a hybrid unstructured layout is an entirely different concept and requires utilization of hanging nodes. In this work, the velocity components normal to the cell faces are located in the middle of the shared cell surfaces while the pressure is stored at the cell centers. Even though for every cell the pressure is stored at its center, the velocity components to be calculated are located in the middle of the shared faces and their number depend on the level of refinement of the adjacent cells, as shown in Fig. 3 . This corresponds to an unstructured staggered grid layout; however, the velocity field is reconstructed and defined at the cell centers, as well, as in the collocated grids. Hence, one cell may be governed by 6 to 24 normal velocity components. The hybrid formulation enables the satisfaction of both the continuity equation and the momentum equations on arbitrarily refined cells, avoiding at the same time odd-even decoupling of the pressure nodes.

Even though fully unstructured grid arrangement is adopted in this work, for clarity we assume that a computational cell adopt a locally structured grid indexing system $(i, j, k)$, as shown in Fig. 3 . The continuity equation (Eq. 11) is discretized by integrating it over control volumes of the unstructured grid. If we assume that the total number of the shared faces of each side $\left(l \pm \frac{1}{2}\right),(l=i, j, k)$ of a reference cell, $p$, is $\left.N_{F a c e s}\right|_{l \pm \frac{1}{2}}$, the discrete divergence operator over the cell will be:

$$
\mathcal{D}(\mathbf{u})=\operatorname{vol}\left(\frac{\sum_{N=1}^{N_{\text {Faces }} \mid i+\frac{1}{2}}\left(u_{1}\right)_{\left(i+\frac{1}{2}\right)_{N}} S_{\left(i+\frac{1}{2}\right)_{N}}-\sum_{N=1}^{N_{\text {Faces }} \mid i-\frac{1}{2}}\left(u_{1}\right)_{\left(i-\frac{1}{2}\right)_{N}} S_{\left(i-\frac{1}{2}\right)_{N}}}{\Delta x \sum_{N=1}^{N_{\text {Faces }} \mid i+\frac{1}{2}} S_{\left(i+\frac{1}{2}\right)_{N}}}+\ldots\right)=0
$$

where $v o l$ is the control volume, $S_{\left(l \pm \frac{1}{2}\right)_{N}}, N=1,\left.N_{\text {Faces }}\right|_{l \pm \frac{1}{2}}$ represent the area of the shared faces and $\mathbf{u}_{\left(l \pm \frac{1}{2}\right) N}$ the corresponding face centered Cartesian velocity components. 
As mentioned above, to avoid odd-even decoupling of the pressure nodes and facilitate the discretization procedure, the normal to the cell faces velocity components are calculated in the middle of the shared faces of the unstructured mesh and the three velocity components are then reconstructed at the cell centers. The velocity field can be calculated at the cell centers from the cell face Cartesian velocity components by averaging the corresponding volume fluxes and dividing by the corresponding normal surface area. For instance, the reconstruction of the Cartesian velocity component $\left(u_{1}\right)$ is accomplished as follows:

$$
\left(u_{1}\right)=\frac{\sum_{N=1}^{N_{\text {Faces }} \mid i+\frac{1}{2}}\left(u_{1}\right)_{\left(i+\frac{1}{2}\right) N} S_{\left(i+\frac{1}{2}\right)_{N}}+\sum_{N=1}^{N_{\text {Faces }} \mid i-\frac{1}{2}}\left(u_{1}\right)_{\left(i-\frac{1}{2}\right) N} S_{\left(i-\frac{1}{2}\right)_{N}}}{2 \sum_{N=1}^{N_{\text {Faces }} \mid i-\frac{1}{2}} S_{\left(i-\frac{1}{2}\right)_{N}}}
$$

and similarly the $\left(u_{2}\right),\left(u_{3}\right)$ components are determined by interpolating along their perspective Cartesian directions. Having calculated the Cartesian velocity components at the cell centers, discretization schemes can be implemented in the same manner as in collocated grids, as explained below.

The momentum equations (2) can be formulated in a discrete operator form as follows:

$$
\frac{\partial u_{j}}{\partial t}=-\mathcal{A}\left(u_{j}\right)-\mathcal{G}_{j}(p)
$$

where $\mathcal{G}$ is the pressure gradient operator and $\mathcal{A}$ is a summation of the convective $\mathcal{C}$ and viscous $\mathcal{V}$ operators:

$$
\mathcal{A}(.)=C(.)-\frac{1}{\operatorname{Re}} \mathcal{V}(.)
$$

51 which are defined as:

$$
C(.)=\frac{\partial}{\partial x_{i}}\left(u_{i}\right)
$$

and

$$
\mathcal{V}(.)=\frac{\partial}{\partial x_{i}}\left(\frac{\partial}{\partial x_{i}}\right)
$$

respectively. The pressure gradient operator is calculated by using the differencing operator $\delta_{\xi}$, defined in (11), as follows:

$$
\mathcal{G}_{j}(p)=\delta_{x_{j}}(p)
$$

For instance, discretizing the $x$ momentum equation in the middle of the $N=1,\left.N_{\text {Faces }}\right|_{i+\frac{1}{2}}$ shared faces of the unstructured mesh (Fig. 3, we obtain the following semi-discrete form at $\left(i+\frac{1}{2}\right)$ :

$$
\left.\frac{\partial u_{1}}{\partial t}\right|_{\left(i+\frac{1}{2}\right)_{N}}=-\left.\mathcal{A}\left(u_{1}\right)\right|_{\left(i+\frac{1}{2}\right)_{N}}-\left.\mathcal{G}_{1}(p)\right|_{\left(i+\frac{1}{2}\right)_{N}}
$$

256 The $\left.\mathcal{G}_{1}(p)\right|_{\left(i+\frac{1}{2}\right)_{N}}$ term is the differencing operator (Eq. 11 applied to calculate the pressure gradient at the faces. Essentially, the 2D Lagrangian operator (Eq. 7) is used to calculate the pressure values at hanging nodes, from cell centered values
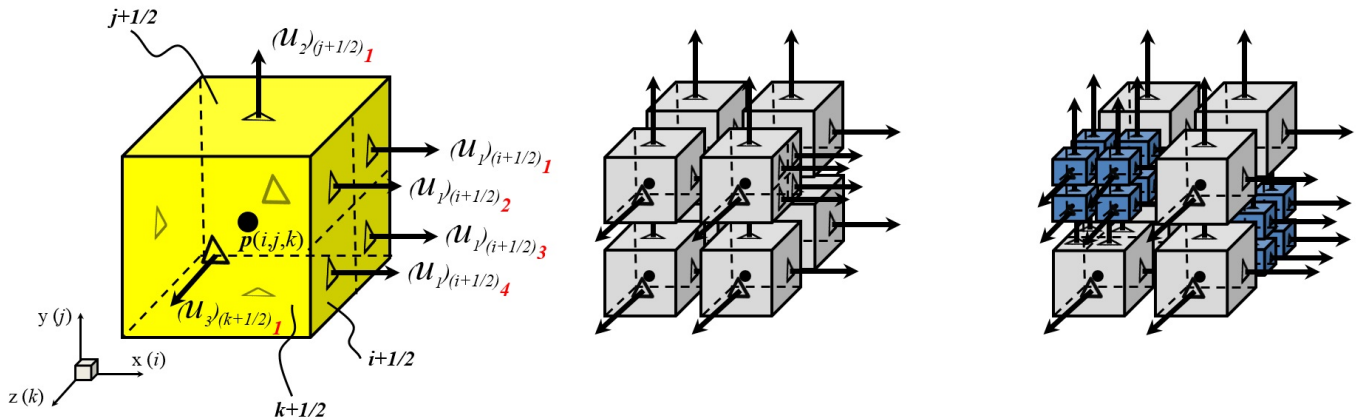

Fig. 3: Hybrid staggered/non-staggered grid layout on unstructured Cartesian grids. • Cell centered values of pressure or the reconstructred velocity components; $\triangle$ face centered normal velocity components. 

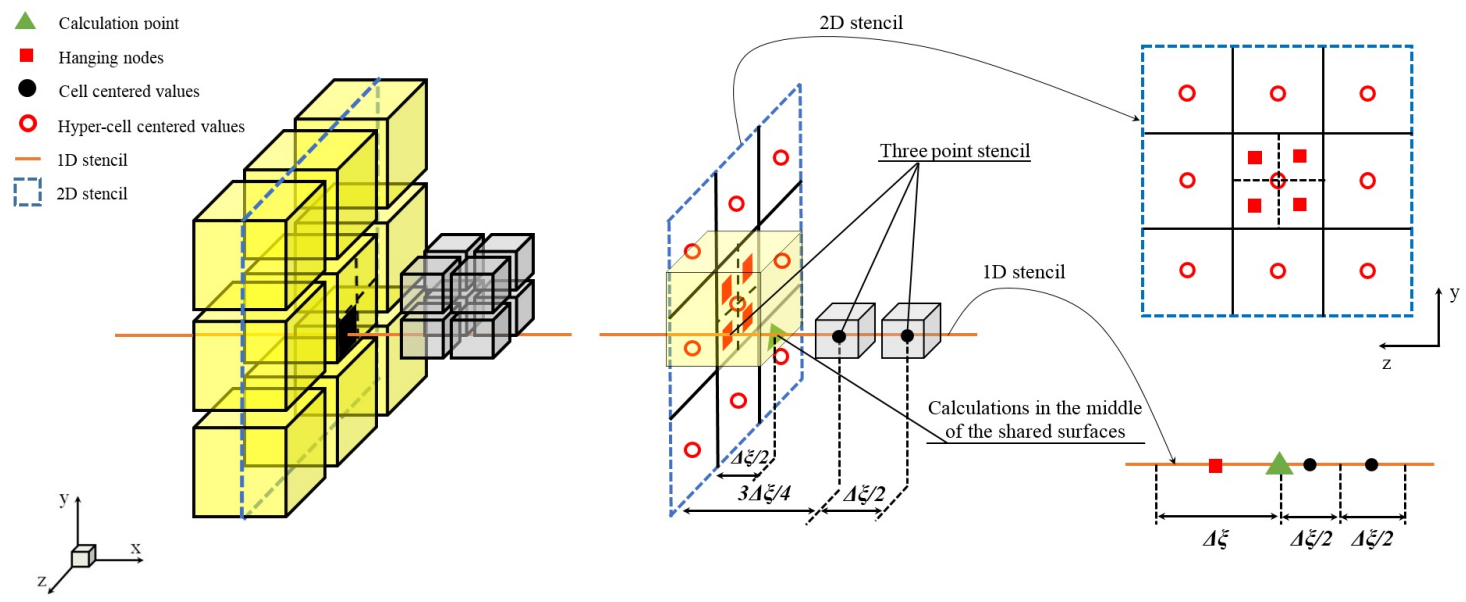

Fig. 4: Utilization of hanging nodes and construction of 1D and 2D stencils of collinear or coplanar nodes, respectively, to facilitate the finite difference calculations.

of the hyper-cells, and subsequently, a second order accurate derivative is calculated on a three-point stencil of collinear points (Eq. 11). In this case, the hanging nodes are located at the normal intersection of a line passing from the center of the finer cell and a plane passing from the center of mass of hyper-cells, that constitute a $3 \times 3$ stencil which is parallel to the calculation face, as shown in Fig. 4 . Additionally, assuming that the $\mathcal{A}_{i, j, k}$ terms have been calculated at all the cell centers, the above mentioned three-point stencil can also be utilized to perform 1D Lagrange interpolation (Eq. 4) and calculate the terms $\mathcal{A}$ at the perspective surfaces. Whenever the adjacent cells have equal grid resolution, central differences are applied and no hanging nodes are needed. In such a case, reconstruction of the flow variables from the cell faces to their centers of mass and the opposite is obtained by averaging the corresponding values [77].

Concerning the spatial discretization of the convective terms in the momentum equation, we are using the conventional cell-centered based second-order central differencing scheme (CDS) when the support stencil includes cells of equal grid resolution. This approach has been successfully utilized in the CURVIB solver where the hybrid staggered/non-staggered was adopted [25, [12, 77]. However, to control the level of accuracy when discretizing the governing equations around arbitrarily refined cells, hanging nodes are used and the conventional second-order central differencing scheme is modified. To calculate the convective flux of a dependent variable, $\phi$, in the middle of shared surfaces from cells with varying grid resolution, we use a 1D three-point stencil to perform quadratic interpolation (Fig. 5). By doing so, the convected variable $\phi$ is calculated on a non-uniform stencil with second order accuracy and strong coupling between coarse/fine interfaces is achieved. Figure 5 shows 1D analysis of the discretization scheme employed when a reference cell, $i$, is adjacent to an isotropically refined cell. In this case, since the value of the dependent variable $\phi$ cannot be calculated at $i+1 / 2$ by using a uniform stencil, three-point quadratic interpolation is used. The 1D support stencil can be chosen accordingly to reach the desired level of accuracy and robustness. For instance, second-order upwind scheme is used around cells of varying level of refinement when eddy resolving methods are employed.

The convection term (Eq. 16) of a variable $\phi$ for the first velocity component $u_{1}$, for example, is discretizated on a hybrid staggered/non-staggered layout when using a second-order central differencing scheme, as follows:

$$
\left.C(\phi)\right|_{i+\frac{1}{2}}=\left.\frac{1}{2} \frac{\partial}{\partial \xi}\left(u_{1} \phi\right)\right|_{i}+\left.\frac{1}{2} \frac{\partial}{\partial \xi}\left(u_{1} \phi\right)\right|_{i+1}
$$

with

$$
\left.\frac{\partial}{\partial \xi}\left(u_{1} \phi\right)\right|_{i}=\left(u_{1}\right)_{i+\frac{1}{2}} \frac{\phi_{i+1}+\phi_{i}}{2 \Delta \xi}-\left(u_{1}\right)_{i-\frac{1}{2}} \frac{\phi_{i}+\phi_{i-1}}{2 \Delta \xi}
$$

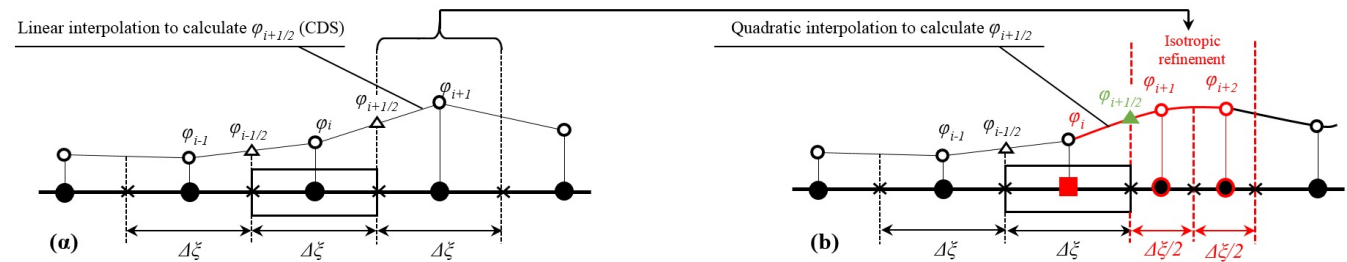

Fig. 5: One dimensional representation of the discretization scheme employed when a reference cell is adjacent to other cells of a) Equal and b) Varying refinement levels. • Cell centered values; cell centered values (1D) or values at hanging nodes (2D or 3D); $\Delta$ calculations in the middle of the shared surfaces between neighboring cells of varying refinement levels. 
In the general case when a cell $i$ of level $\mathrm{L}^{i}$ and grid spacing $\Delta \xi$ is adjacent to the refined cells $i+1$ and $i+2$ of level $\mathrm{L}^{i+1}$ and grid spacing $\frac{1}{2} \Delta \xi$, the semi-discrete convective term is calculated as follows:

$$
\left.C(\phi)\right|_{i+\frac{1}{2}}=\left.\frac{\partial}{\partial \xi}\left(u_{1} \phi\right)\right|_{i} L_{0}+\left.\frac{\partial}{\partial \xi}\left(u_{1} \phi\right)\right|_{i+1} L_{1}+\left.\frac{\partial}{\partial \xi}\left(u_{1} \phi\right)\right|_{i+2} L_{2}
$$

where $L=L_{k}(\xi), k=0,2$ the Lagrange basis polynomials (Eq. 3) which apply quadratic interpolation on the three-point stencil. The cell centered convective terms for the cell $i$, is calculated as:

$$
\left.\frac{\partial}{\partial \xi}\left(u_{1} \phi\right)\right|_{i}=\frac{\left(u_{1}\right)_{i+\frac{1}{2}} \phi_{i+\frac{1}{2}}-\left(u_{1}\right)_{i-\frac{1}{2}} \phi_{i-\frac{1}{2}}}{\Delta \xi}
$$

The values of $\phi_{i-\frac{1}{2}}$ and $\phi_{i+\frac{1}{2}}$ are determined with linear interpolation from the neighboring cells if they have the same resolution and can form uniform stencil or with quadratic interpolation otherwise. For instance, if the cells $i-2, i-1, i$ have the same refinement level, $\mathrm{L}^{i}$ and the cells $i+1$ and $i+2$ are refined cells with $\mathrm{L}^{i+1}$ (Fig. 5) we get:

$$
\left.\frac{\partial}{\partial \xi}\left(u_{1} \phi\right)\right|_{i}=\frac{\left(u_{1}\right)_{i+\frac{1}{2}}\left(L_{0} \phi_{i}+L_{1} \phi_{i+1}+L_{2} \phi_{i+2}\right)-\left(u_{1}\right)_{i-\frac{1}{2}}\left(\frac{1}{2} \phi_{i}+\frac{1}{2} \phi_{i-1}\right)}{\Delta \xi}
$$

Apparently, this approach extends the size of the overall stencil from three-point, which corresponds to the second-order central scheme, to four- or five-point stencil, depending on the grid resolution of the adjacent cells. The implementation of the described discretization scheme around multi-resolution cells is straightforward to 2D or 3D grids by calculating the value of the flow variable $\phi$ at hanging nodes in order to form the 1D stencil. The location and the calculation of values at the hanging nodes involved in the calculation of the convective terms have been described above where we presented the calculations normal to the corresponding cell faces (Fig. 4).

To calculate the viscous terms $\mathcal{V}$ (Eq. 17), we extend herein the above mentioned approach and use hanging nodes, Lagrange interpolation (Eqs. (4), (7p) and the second order differencing operator of Eq. (11). The viscous terms are calculated at the cell centers and subsequently interpolated in the middle of the shared surfaces, procedure described above. The semi-discrete cell centered viscous term for the $\left(u_{1}\right)$ component of the momentum equation can be written as:

$$
\left.\mathcal{V}\left(u_{1}\right)\right|_{i, j, k}=\frac{\partial}{\partial x_{i}}\left(\frac{\partial}{\partial x_{i}}\left(u_{1}\right)\right)=\frac{\left.\frac{\partial u_{1}}{\partial x_{1}}\right|_{i+\frac{1}{2}, j, k}-\left.\frac{\partial u_{1}}{\partial x_{1}}\right|_{i-\frac{1}{2}, j, k}}{\Delta x_{1}}+\frac{\left.\frac{\partial u_{1}}{\partial x_{2}}\right|_{i, j+\frac{1}{2}, k}-\left.\frac{\partial u_{1}}{\partial x_{2}}\right|_{i, j-\frac{1}{2}, k}}{\Delta x_{2}}+\frac{\left.\frac{\partial u_{1}}{\partial x_{3}}\right|_{i, j, k+\frac{1}{2}}-\left.\frac{\partial u_{1}}{\partial x_{3}}\right|_{i, j, k-\frac{1}{2}}}{\Delta x_{3}}
$$

and similarly the $\mathcal{V}\left(u_{2}\right), \mathcal{V}\left(u_{3}\right)$ components are obtained. Here it is implied that central differences are used to calculate the second derivatives at the cell centers, using the face centered first derivatives. The first derivatives are calculated in the middle of the shared cell faces by using the differencing operator of Eq (11).

\subsection{Time integration method}

The fractional step algorithm is outlined as follows. The momentum equations are solved implicitly discretized in time via the second order accurate backward Euler differencing scheme:

$$
\frac{3 \mathbf{u}^{*}-4 \mathbf{u}^{n}+\mathbf{u}^{n-1}}{2 \Delta t}=-\frac{\nabla p^{n}}{\rho}-\mathbf{u}^{*} \nabla \mathbf{u}^{*}+\frac{1}{R e} \nabla^{2} \mathbf{u}^{*}
$$

where $n$ denotes the time step and $\mathbf{u}^{*}$ represents the intermediate staggered velocity field, which does not satisfy the continuity equation. The right hand side of the above equation is discretized in space according to the hybrid staggered/nonstaggered approach described. The pressure correction step is employed to enforce satisfaction of the continuity equation at the time step $n+1$ :

$$
\begin{gathered}
-\frac{3 \rho\left(\mathbf{u}^{n+1}-\mathbf{u}^{*}\right)}{2 \Delta t}=\nabla \Pi^{n} \\
\nabla \mathbf{u}^{n+1}=0
\end{gathered}
$$

308 and the pressure correction, $\Pi$ :

$$
\Pi=p^{n+1}-p^{n}
$$

can be obtained by the Poisson equation resulted by the combination of the Eqs. 27] and (28):

$$
\nabla^{2} \Pi=\frac{3 \rho \nabla \mathbf{u}^{*}}{2 \Delta t}
$$


310

The velocity field that satisfies the continuity equation is then obtained as follows:

$$
\mathbf{u}^{n+1}=\mathbf{u}^{*}-\frac{2 \Delta t \nabla \Pi}{3 \rho}
$$

311

312

The Jacobian-free Newton-Krylov method is employed to solve the momentum equation in a fully implicit manner. The discretization of pressure (Eq. 18) following the strong coupling approach, leads to the generation of a non-symmetric sparse matrix. For this reason, the Algebraic Multi-Grid (AMG) serves as the preconditioner to accelerate the convergence rate of the Newton-Krylov solver when solving the Poisson equation (Eq. 30.

\section{Sharp interface immersed boundary method on adaptively refined meshes}

We adopt herein the sharp-interface immersed boundary of [12] to handle arbitrarily complex stationary or moving bodies. The key new feature of our work is that local grid refinement of the background mesh, in the vicinity of immersed boundaries improves the accuracy of the calculations with low computational cost. Algorithmically, the sharp interface immersed boundary method involves two steps: a) The grid node classification and b) the reconstruction of the velocity and pressure fields and imposition of appropriate boundary conditions in the immediate vicinity of the immersed body [26, 12].

According to the present approach, the governing equations are discretized on a background grid which does not conform to the physical boundaries of the immersed bodies. For this reason, the cell centered nodes of the background unstructured mesh are classified as either solid, IB (immersed boundary) or fluid nodes, as required by the sharp-interface immersed boundary method. The solid nodes are the nodes which are located inside the body and are excluded from the calculations. The IB nodes are in the immediate vicinity but exterior to the body and need special treatment in order to impose the appropriate boundary conditions in the vicinity of the complex boundaries (see below). The governing equations are discretized and solved on all the other nodes of the background grid which are classified as fluid nodes (Fig. 6a). To identify the solid nodes on an arbitrarily refined mesh, the immersed boundaries are discretized using unstructured triangular meshes and the ray-casting method is employed. The overall search algorithm is accelerated by adopting the concept of the bounding box, proposed by Borazjani et al. [12].

Having identified the IB nodes, the pressure (if the body is moving) and the velocity field need to be reconstructed in order to impose the appropriate boundary conditions in immediate the vicinity of the immersed body. Essentially we extend the approach initially proposed by [26] who discretized the governing equations around immersed boundaries on a hybrid staggered/non staggered structured grid layout and used a second-order accurate dual-time-stepping artificial compressibility iteration scheme. If we assume that the dynamically evolving solid surface $\Gamma^{b}(t)$ is represented by a set of $K$ material points that lie on $\Gamma^{b}(t)$, the corresponding Lagrangian position vector $\mathbf{r}^{k}(t)$ will be:

$$
\mathbf{r}^{k}(t) \in \Gamma^{b}(t) \quad \forall \quad t>0, \quad \text { with } \quad \mathbf{r}^{k}(0)=\mathbf{r}_{0}^{k} \quad \forall \quad k=1, K
$$

with $\mathbf{r}_{0}^{k}$ being the initial position of the $k^{\text {th }}$ material point on $\Gamma_{0}^{b} \equiv \Gamma^{b}(0)$. In fact, the material points are the nodes of the triangular mesh of the immersed body. The prescribed motion of the immersed body correlate the velocity of the material points with the temporal variation of the location of those points; as a result of the movement of the interface $\Gamma^{b}(t)$ :

$$
\frac{\partial \mathbf{r}^{k}}{\partial t}=\mathbf{U}^{k}(t), \quad \text { with } \quad \mathbf{r}^{k}(0)=\mathbf{r}_{0}^{k} \quad \forall \quad k=1, K
$$

The Lagrangian specification of the velocity of the material points can be linked with the Eulerian velocity vector:

$$
\mathbf{u}\left(\mathbf{r}^{k}(t), t\right)=\mathbf{U}^{k}(t) \quad \forall \quad k=1, K
$$

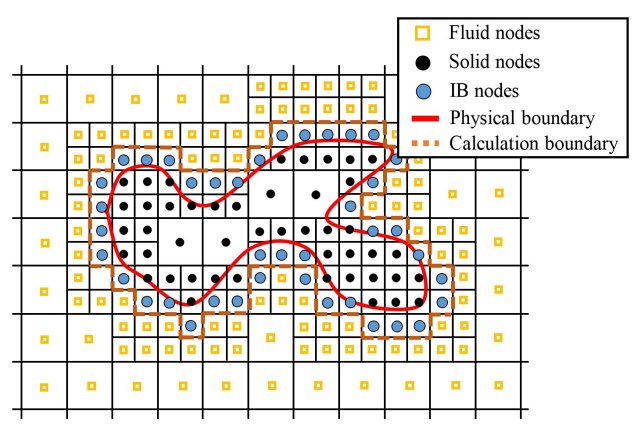

(a) Classification of the nodes of the unstructured mesh

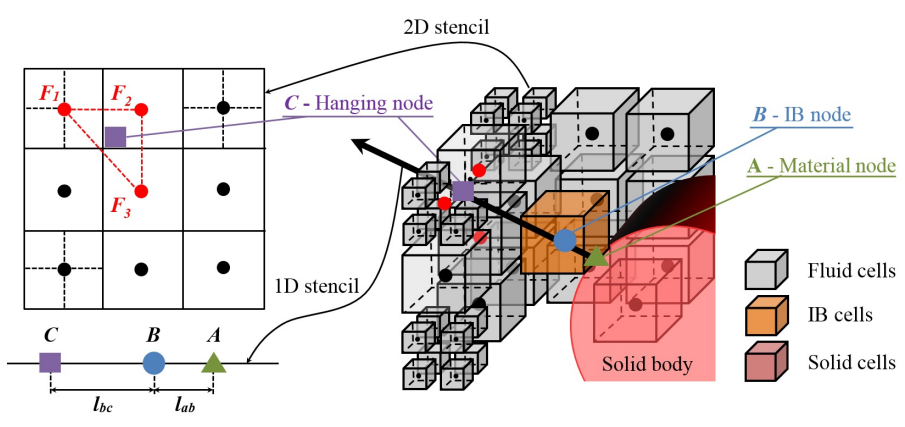

(b) Utilization of stencils to reconstruct the pressure and velocity field around the immersed bodies on unstructured Cartesian grids.

Fig. 6: Classification of the cell centered nodes on unstructured Cartesian grids and utilization of 1D three-point stencils to facilitate the reconstruction of pressure and velocity field around immersed bodies. 
and this constitutes boundary condition to be imposed at every time step.

The pressure field around the immersed body can be calculated by enforcing the Neumann boundary condition on the $\Gamma^{b}(t)$ interface, by projecting the momentum equation along to the wall normal direction, $\Gamma^{b}$ and neglecting the viscous forces and the subgrid-scale stresses:

$$
-\left.\frac{\partial p}{\partial n}\right|_{\mathbf{r}^{k}}=\mathbf{n}^{k} \frac{\partial \mathbf{U}^{k}(t)}{\partial t}
$$

where $\mathbf{n}^{k}$ denotes a unit vector normal to the body at $\mathbf{r}^{k}$. Note that the pressure on the immersed boundary surface is required when the forces imparted by the flow on the body need to be calculated.

Concerning the discretization of the governing equations in the immediate vicinity of the immersed body, we extend the approach described by [26, 12] on unstructured Cartesian grids. Initially, the velocity field is reconstructed at all the IB nodes (node- $B$ ) from the material nodes (node- $A$ ) and the hanging nodes (node- $C$ ) (Fig. 6b). Nodes-A correspond to each IB nodes (node- $B$ ) and are identified by projecting the IB nodes to the IB surface triangular mesh. Dirichlet condition is imposed at the nodes- $A$ and the components of the velocity field are calculated from the values of the material points (the vertices of the triangular elements) from Eq. [34, by using an inverse distance function [26]. The hanging nodes, node- $C$, are identified as the closest intersection of the surface-normal vector and any of the six planes around the IB cells, which are essentially $3 \times 3$ support stencils formed from the cell centered values of the surrounding hyper-cells, and are parallel to the IB cells' faces (Fig. 2). Then, having identified the $3 \times 3$ support stencil (see Appendix A), the three closest, coplanar hyper-cell centers $\left(F_{1}, F_{2}\right.$ and $\left.F_{3}\right)$ are used to calculate the velocity components at the nodes- $C$, by using the above mentioned inverse distance function, as shown in Fig. 6b. Finally, the velocity components at the IB nodes are calculated from the nodes- $A$ and $C$ as described in [27]. Since we have adopted a staggered/non-staggered grid layout, the velocity components are also updated at the fluid/IB cell faces by performing linear interpolation between the corresponding cells. The velocity field prescribed at the fluid/IB cell faces, lying in the vicinity of the immersed body, constitute boundary conditions for the momentum equations (Fig. 6a). The global mass around the immersed bodies is fully conserved after performing integration and correction of the fluxes; by doing so the Navier-Stokes and continuity equations constitute a well posed problem [32]. The pressure field needs to be reconstructed at the IB nodes, $p_{b}$, only if the immersed body is moving, due to the hybrid staggered/non-staggered layout. In such a case, the pressure at the IB nodes can be obtained after discretizing Eq. (35) and enforcing Neumann condition:

$$
p_{b}=p_{c}+\mathbf{n} \frac{\mathbf{U}^{n}-\mathbf{U}^{n-1}}{\Delta t} l_{b c}
$$

where $l_{c b}$ is the distance between the nodes- $A$ and $B$ (Fig. 6b), the superscripts $n$ and $n-1$ denote the current and previous time steps and $p_{c}$ is the value for pressure at node- $C$ which can be calculated following the above mentioned approach to reconstruct the velocity field.

\section{Results and discussion}

\subsection{Solution of a Poisson-like problem on unstructured grids}

In order to investigate the accuracy of the Poisson solver we consider herein a Poisson equation:

$$
\nabla^{2} \varphi=S(x, y)
$$

with the following source term:

$$
\begin{array}{r}
S(x, y)=-100 A e^{-B}+50^{2} x^{2} A e^{-B}+50^{2} y^{2} e^{-B} A-500 \pi x A e^{-B} \cos (5 \pi x) \sin (5 \pi x) \\
-500 \pi y A e^{-B} \sin (5 \pi y) \cos (5 \pi y)-50 \pi^{2} e^{-B}
\end{array}
$$

\begin{tabular}{|c|c|c|c|c|c|c|c|c|c|c|c|c|}
\hline \multirow[b]{2}{*}{ Primary grid } & \multicolumn{4}{|c|}{ Number of grid nodes } & \multicolumn{4}{|c|}{$L_{\infty}=\max \left|\varphi-\varphi_{\text {exact }}\right|$} & \multicolumn{4}{|c|}{$E_{h}=\left|\|\nabla \varphi\|^{2}-\left\|\nabla \varphi_{\text {exact }}\right\|^{2}\right|^{1 / 2}$} \\
\hline & $11 \times 11$ & $21 \times 21$ & $41 \times 41$ & $81 \times 81$ & $11 \times 11$ & $21 \times 21$ & $41 \times 41$ & $81 \times 81$ & $11 \times 11$ & $21 \times 21$ & $41 \times 41$ & $81 \times 81$ \\
\hline No refinement & 100 & 400 & 1600 & 6400 & 1.76539 & 0.21195 & 0.05560 & 0.01250 & 3.89682 & 1.10254 & 0.59504 & 0.28907 \\
\hline 1 Level & 196 & 736 & 2944 & 11812 & 0.21247 & 0.05575 & 0.01254 & 0.00326 & 1.10415 & 0.59567 & 0.28917 & 0.14522 \\
\hline 2 Levels & 340 & 1552 & 7072 & 28732 & 0.05734 & 0.01256 & 0.00330 & 0.00082 & 0.60533 & 0.29311 & 0.14564 & 0.07272 \\
\hline 3 Levels & 580 & 3904 & 19864 & 79780 & 0.02003 & 0.00367 & 0.00090 & 0.00024 & 0.40591 & 0.15444 & 0.07366 & 0.03682 \\
\hline
\end{tabular}

and $A=\sin (5 \pi x) \sin (5 \pi y), B=25 x^{2}+25 y^{2}$. This equation has an exact solution governed by a circular Gaussian function and sinusoidal amplitude modulation, as follows:

$$
\varphi(x, y)=A \exp \left(-\left(\left(x-x_{0}\right)^{2}+\left(y-y_{0}\right)^{2}\right) / 2 \sigma^{2}\right)
$$




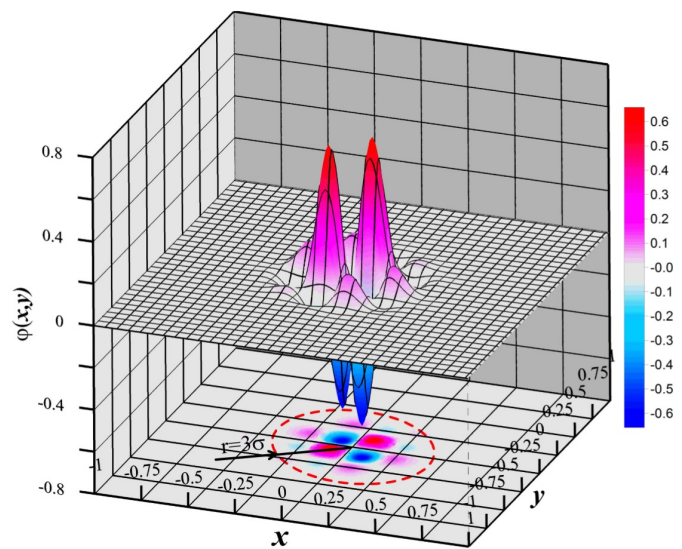

Fig. 7: The exact solution of a Poisson equation, governed by a circular Gaussian function and a sinusiudal amplitude modulation (Eq. 39.

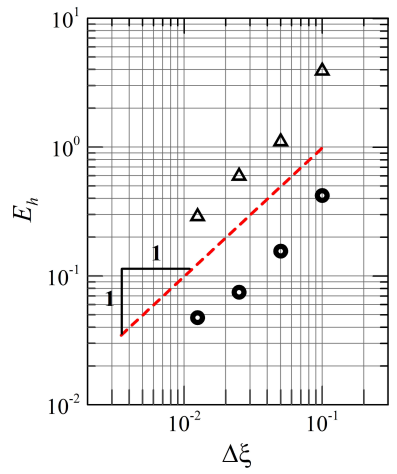

(a) Average energy of the error, $E_{h}$, as a function of the grid spacing.

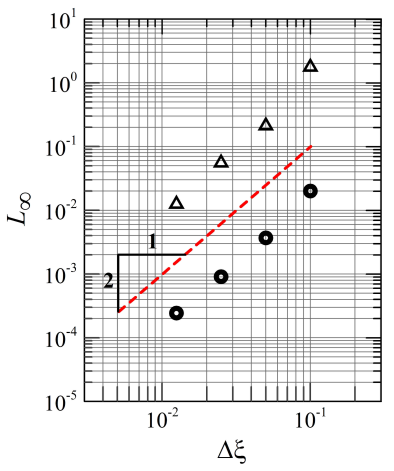

(b) $L_{\infty}$ error, as a function of the grid spacing.

Fig. 8: Log-log graphs of the average energy of the error, $E_{h}$, and the $L_{\infty}$ error, as a function of the spacing of the corresponding primary uniform Cartesian grid; $\circ 3$ level refinement; $\Delta$ uniform grid.

The center of the above distribution is $\left(x_{0}, y_{0}\right)=(0,0)$ and the standard deviation is $\sigma=\frac{1}{\sqrt{50}}$; the numerical solution in the domain $\Omega^{2}:[-1: 1] \times[-1: 1]$ can be derived by applying Neumann boundary conditions.

The solution derived by solving Eq. (37) numerically on successively finer uniform grids with $11^{2}, 21^{2}, 41^{2}, 81^{2}$ and $161^{2}$ nodes, is compared with numerical solutions obtained by applying three levels of refinement on the above uniform Cartesian meshes. In all the locally refined computations the highest level of grid refinement is adapted within a circle with a radius $\rho=3 \sigma$; that is, the refined zone covers the region where the solution of Eq. (37) exhibits steep and oscillatory gradients, as shown in Fig. 7. Table 1 shows the reduction of $L_{\infty}$ error norm and the average error in energy, $E_{h}$, by increasing the resolution of the primary grid and/or applying adaptive local refinement - all the errors are computed in terms of the analytical solution given by Eq. (39). It can be observed that not only the error in all the calculations is reduced with increasing the levels of refinement but equivalent grid resolution, within the refined zone, results to comparable prediction of the error (e.g. uniform Cartesian grid $41 \times 41$ versus locally refined $11 \times 11$ grid with 2 levels or refinement). However, the ellipticity of the problem is associated with the fact that the error of a refined grid is always higher when compared to that of a uniform grid with equivalent maximum discretization.

The current study also reveals the order of accuracy of the proposed methodology; in Figs. $8 \mathrm{a}$, 8b the reduction of the $L_{\infty}$ and the average energy error $E_{h}$ are plotted, respectively, as a function of the grid spacing of the primary grid. It may be observed that the convergence rate in the energy norm is $O\left(h^{1}\right)$ and in the $L_{\infty}$ error norm is $O\left(h^{2}\right)$ as expected. The latter $\log -\log$ graphs reveal that the current approach is $2^{\text {nd }}$ order accurate since the slope in the $L_{\infty}$ is -2 and in the $E_{h}$ is - 1 . The performance of the grid refinement approach is demonstrated by plotting the distribution of the energy of the error as a function of the levels of refinement, while keeping the primary grid resolution constant (Fig. 9). It may be noticed that the local increase of the level of refinement is accompanied with a monotonic reduction of the energy norm. We also note that in all calculations the strongly coupled discretization enabled convergence to machine zero.

\subsection{Laminar flow past a sphere}

Laminar flow separation and vortex shedding past a sphere has been a widely used as a test case to validate incompressible flow solvers using immersed boundary methods [27, 26, 69]. The presence of thin boundary layers in the accelerating
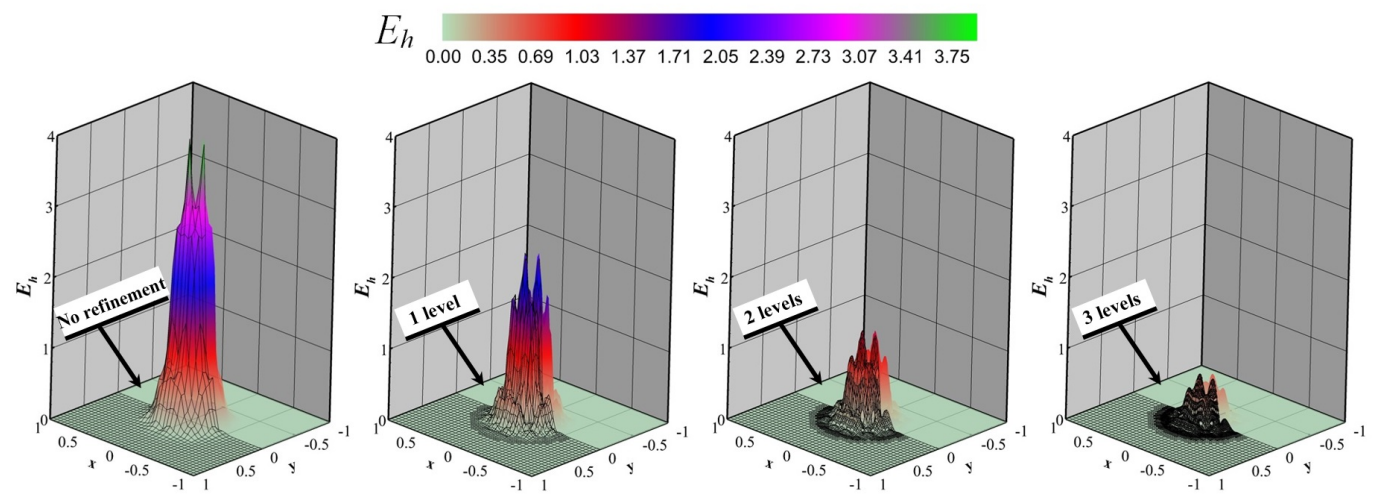

Fig. 9: Distribution of the energy of the error calculated by solving the Poisson equation on a primary uniform Cartesian grid $41 \times 41$ and after applying 1-3 levels of refinement. 
portion of the flow and the sensitivity of the predicted wake structure to the prediction of the separation point off the sphere surface make this an ideal case for validating the locally refined unstructured Cartesian immersed boundary method. Based on the majority of previous benchmark numerical [40, 31, 68] and experimental [39, 75] studies, the flow at Reynolds number between $20<R e<210$ is separated, steady and axisymmetric. The flow remains steady but loses axial symmetry for $210<\operatorname{Re}<270$ [38] while the loss of axisymmetry and the steady double-thread wake was also seen by Nakamura [43] at $R e=190$. The unsteady wake transition has been observed experimentally [39] and numerically [68, 31] at $270<R e<285$ and periodic vortex shedding occurs at $290<R e<400$. Transition from one-frequency flow to almost chaotic system occurs between $R e=300$ and $R e=500$ and increase of symmetry in the average flow is found with increasing the Reynolds number [68].

Our results here are compared against previous experimental measurements [65, 43] and numerical simulations [40, 31, 68] where conforming grids were utilized. Specifically, Taneda [65] and Nakamura [43] studied experimentally the wake of spheres moving in a water tank, while Magnaudet et al. [40] performed computations over the range of $0.1 \leq R e \leq 300$ by discretizing the axisymmetrical Navier-Stokes equations on conforming grids, using a finite-volume approach on a staggered mesh layout. Johnson and Patel [31] studied both the steady and unsteady flow regimes for Reynolds numbers up to 300 and used the method developed by Sotiropoulos and Abdallah [60] to solve the governing equations on a spherical coordinate system. A grid conformed around the sphere was used for all the simulations of Tomboulides and Orszag [68] who implemented a spectral method designed to investigate the flow over three-dimensional axisymmetric geometries and their calculations are found to agree well with previous results of [65, 43, 24, 44].

\subsubsection{Steady flow, $R e=20-200$}

The accuracy of the flow solver is here demonstrated by calculating the steady laminar flow around a sphere, for $20 \leq R e \leq 200$. The Reynolds number is based on the diameter of the sphere, $D$, and the freestream velocity, $U_{\infty}$. A rigid sphere is located at the origin of the Cartesian coordinate system in a rectangular domain extending between $-5 D \leq x \leq 5 D,-5 D \leq y \leq 5 D$ and $-5 D \leq z \leq 10 D$, with $(x)$ being the spanwise, $(y)$ the vertical and $(z)$ the streamwise directions, respectively, resulting in an area blockage ratio of around 8\%o. Uniform inflow is applied at the inlet of the domain, outflow boundary condition is used to model flow at exit and at all the other boundaries of the domain free-slip conditions are imposed. Two grids are initially considered for the Cartesian domain: a primary uniform Cartesian grid $61 \times 61 \times 91$ resulting in 6 cells per sphere diameter and an unstructured grid generated after applying three levels of refinement on the primary grid and in the vicinity of the sphere, resulting in a resolution of 48 cells per sphere diameter and total of $9.5 \times 10^{5}$ computational cells. The grid refinement zones consist of concentric spheres centered at the origin of the coordinate system and their radii are $r_{1}=2 D, r_{2}=1.5 D, r_{3}=D$ corresponding to first, second and third levels respectively. Also, the immersed sphere is represented by a triangular mesh which consists of $1.6 \times 10^{5}$ triangular elements.

In Figs. $10 \mathrm{a}$ and $10 \mathrm{p}$ the results concerning the wake length and the separation angle, measured from the front stagnation point, with respect to the Reynolds number, are shown. It can be concluded that the results obtained from the coarse grid fail to provide accurate prediction of the induced flow field around the sphere. A grid resolution of 6 cells $/ D$ is inadequate to represent the real spherical geometry immersed in the Cartesian grid and predict the flow physics around the sphere. The locally refined grid, with a grid spacing of $\sim 0.021 D$, is shown to be sufficient for yielding calculations in good agreement with earlier published results. In line with previous results, the flow is found to be steady and axisymmetric for $20<R e<200$ and the wake recirculation bubble exists for $R e>20$. It must be noted that even though the recirculation patterns cross different levels of refinement, the prediction of the wake length is accurate. We note that the experimental results from Taneda [65] for $R e>130$ indicate unsteadiness, a trend which is contrary to all more recent experimental observations [75, 39]. The calculated distribution of the pressure coefficient, $C_{P}$, around the sphere for $R e=100$ is

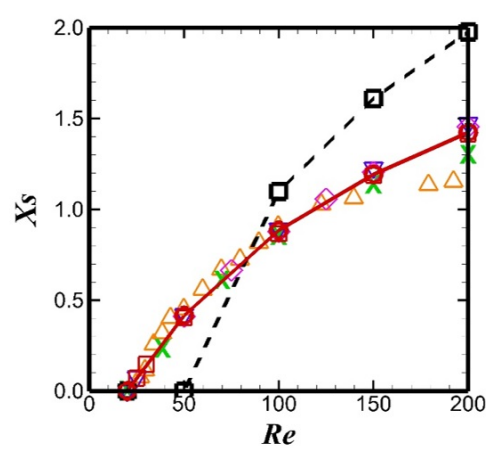

(a)

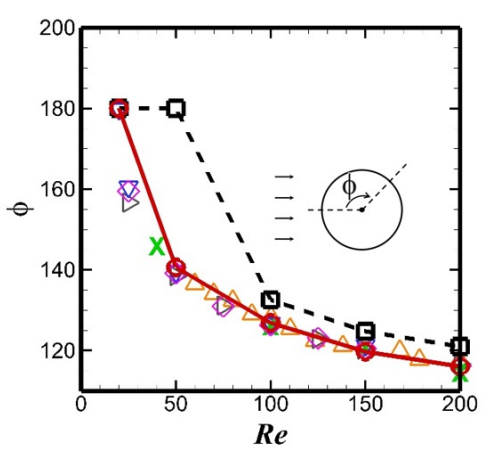

(b)

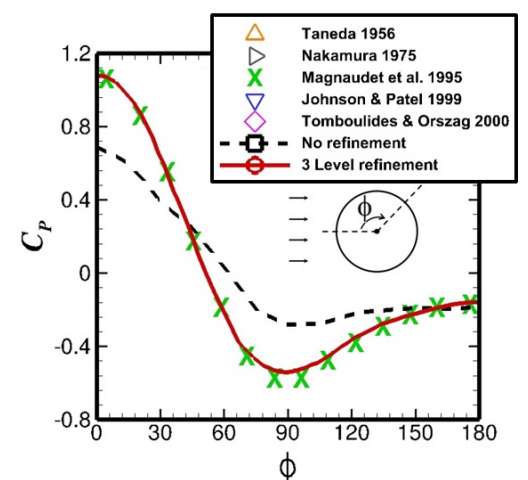

(c)

Fig. 10: Flow past a sphere. (a) Wake length, $X_{s}$ and (b) separation angle, $\phi$, with respect to the Reynolds number, Re. (c) Distribution of the pressure coefficient value, $C_{P}$, along the surface of a sphere at $R e=100$. Comparison of the current calculations performed on a uniform Cartesian grid $61 \times$ $61 \times 91$ without refinement and on a 3-level refined grid adapted around the sphere, against previous experimental measurements 65 [3] and numerical simulations [40, 31 61 68]. 


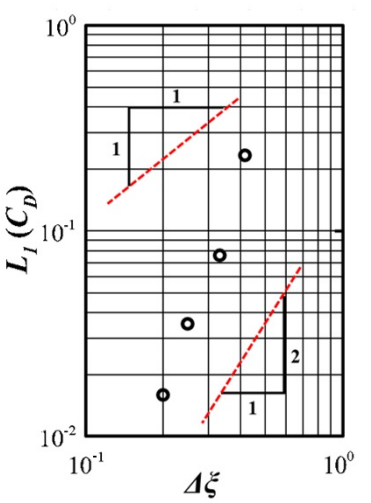

Fig. 11: Variation of the relative error, $L_{1}$, in the predicted drag coefficient, $C_{D}$, as a function of the grid resolution of the primary grid, $\Delta \xi$, by applying 3 levels of refinement around a sphere, at $R e=200$.

$$
\begin{array}{ll}
\longrightarrow & \text { UG - Unstructured grid } \\
-\square- & \text { SG - Uniform structured grid } \\
\longrightarrow & \text { Comparison between UG and SG calculations }
\end{array}
$$

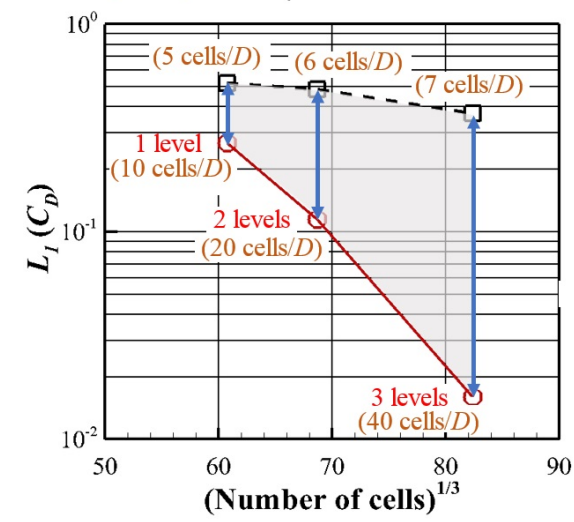

(a)

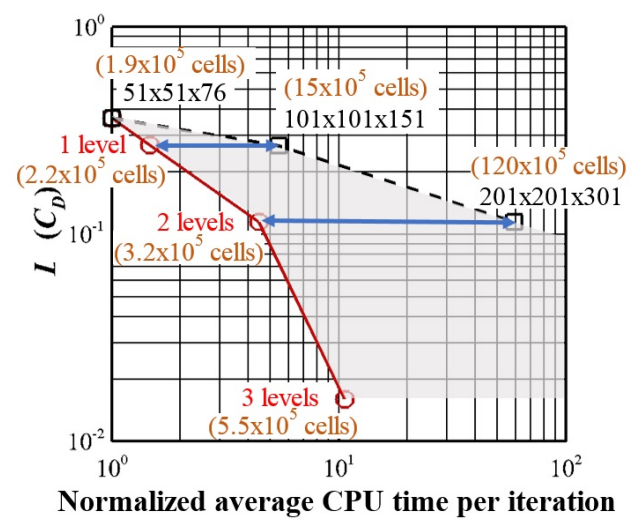

(b)
Fig. 12: Calculation of the relative error, $L_{1}$, in the predicted drag coefficient, $C_{D}$, for the flow past a sphere, at $R e=200$, on grids with (UG) and without (SG) refinement. Comparison between SG and UG calculations of (a) the relative error, $L_{1}$, when using equal number of cells in the computational domain and (b) the average CPU time per iteration when having equal grid spacing in the vicinity of the sphere. The CPU time is normalized with the CPU time of the coarsest grid calculations and a uniform Cartesian grid $51 \times 51 \times 76$ is considered as the primary grid for the local mesh refinement cases.

compared against previous simulations [40]. In this case, the angle is measured from the front stagnation point. While the simulation on the primary coarse grid fails to predict the pressure distribution around the sphere, the results obtained from the refined grid are in very good agreement with the calculations of Magnaudet et al. [40], as shown in Fig. 10k. It should be noted here that the pressure on the surface of the sphere is calculated using first order extrapolation along the local normal to the sphere from the first fluid node, i.e. zero Neumann condition.

Evidence for the second order accuracy of our method is also provided by plotting the variation of the relative error norm, $L_{1}$, of the drag coefficient $L_{1}\left(C_{D}\right)$, as a function of the grid spacing of the primary grid, for $R e=200$ (Fig. 11 ). The exact solution is assumed to be the one obtained on the grid generated after applying three levels of refinement on the $61 \times 61 \times 91$ uniform Cartesian grid. In that case, the calculated value of the drag coefficient is $C_{D}=0.75$ which compares well against previously published data $C_{D}=0.73-0.77$ [53, 35, 40]. The effectivenes of local refinement around the sphere can be further demonstrated by comparing the relative error norm in the predicted drag coefficient with simulations on uniform grids with equivalent number of computational cells. Figure $12 \mathrm{a}$ shows that by increasing the levels of refinement around the sphere, starting from an initially uniform Cartesian mesh $51 \times 51 \times 76$, the relative error of the drag coefficient, $C_{D}$, is decreased monotonically. In all cases, the relative error of the drag coefficient, $L_{1}\left(C_{D}\right)$, is lower compared to calculations performed on uniform Cartesian meshes of equal number of cells. Figure $12 \mathrm{p}$ presents comparison of the relative error, $L_{1}$, and the normalized CPU time between simulations with and without refinement, when using grids with equal spatial resolution in the vicinity of the sphere. It is evident that the CPU time and the computational memory requirements are significantly lower for calculations on unstructured grids, for the same level of accuracy. It should be mentioned that the normalized CPU time is the average CPU time per iteration of the solver normalized with the average CPU time per iteration of the coarsest grid calculations, which was 0.59 seconds performed on 60 CPUs.

\subsubsection{Unsteady flow, $\operatorname{Re}=300$}

The capabilities of the present method to perform accurate unsteady calculations are further illustrated by simulating the flow past a sphere with $R e=300$. For this case the flow is unsteady and asymmetric and the accuracy of the integration of the governing equations in time can also be evaluated. The Reynolds number is based on the diameter of the sphere $D$ and the freestream velocity $U_{\infty}$. A rigid sphere is now located at the origin of the Cartesian coordinate system in a rectangular domain extending between $-5 D \leq x \leq 5 D,-5 D \leq y \leq 5 D$ and $-3 D \leq z \leq 22 D$, with $(x)$ being the spanwise, ( $y$ ) the vertical and $(z)$ the streamwise directions, respectively. As in the previous simulations, the primary grid is $61 \times 61 \times 151$ resulting in 6 cells per sphere diameter. Three levels of refinement are applied to increase the accuracy around the immersed body and in the wake of the sphere. Particularly, the first and second levels of refinement cover spherical domains around the rigid body and have radii equal to $R_{L 1}=2 D$ and $R_{L 2}=1.5 D$, while the mesh refinement is extended in a cylindrical manner from the center of the cylinder up to $21 D$ and $20.5 D$ downstream, respectively (Fig. 13). The third level of refinement is located within an imaginary sphere of radius equal to $R_{L 3}=1 D$. Thus, the unstructured grid consists of $3.2 \times 10^{6}$ cells and the immersed body is represented by a triangular mesh of $1.6 \times 10^{5}$ elements. As a result, the grid spacing close to the sphere is $h=0.02 D$ with $y^{+} \approx 2$. The selected grid spacing is in line with the previous grid sensitivity tests but also agree with the numerical setup of Gilmanov et al. [27] and Vanella et al. [69] who solved the same problem by utilizing a hybrid Cartesian-immersed boundary method and a direct forcing approach, respectively, to treat the immersed body. The time 


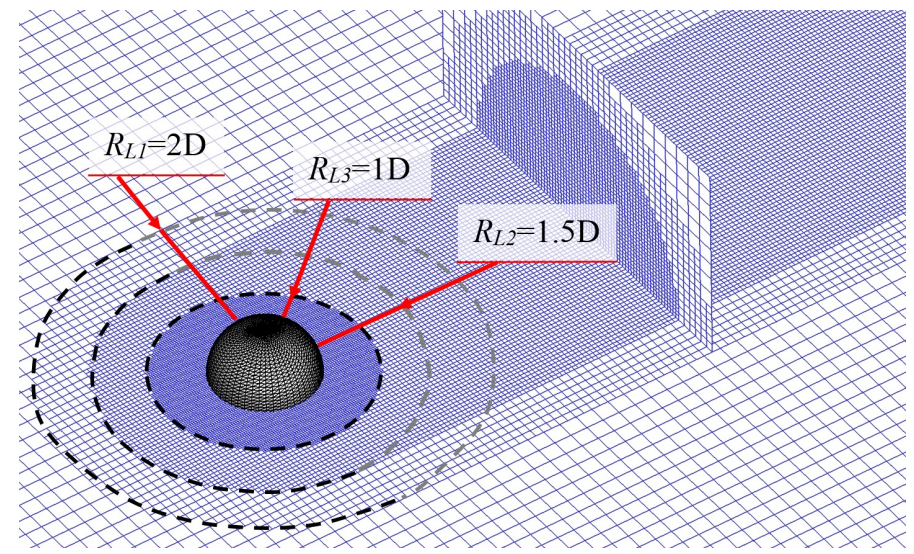

Fig. 13: 3 levels of grid refinement adapted around and in the wake of a stationary sphere; primary uniform Cartesian grid $61 \times 61 \times 91$.
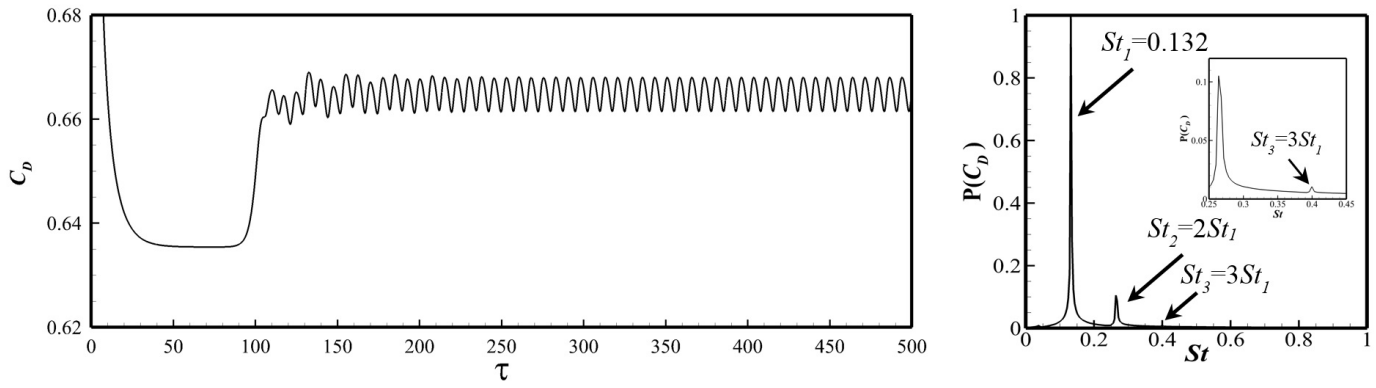

Fig. 14: Time history and single-sided spectrum of the drag coefficient, $C_{D}$, for the flow past a sphere at $R e=300$. Calculations performed on a 3-level refined grid consisted of $3.2 \times 10^{6}$ computational cells.

step chosen for this simulation corresponds to Courant number equal to 0.5 .

At a Reynolds number of $R e=300$, the simulated unsteady flow is periodic and involves asymmetric vortex shedding, in agreement with previous experimental [54, 55] and numerical [40, 31, 68, 50, 69] studies. Figure 14] shows a plot of the drag coefficient, $C_{D}$, versus time and its power spectrum. The dominant frequency is associated with the shedding characteristic frequency and corresponds to the Strouhal number which is $S t=0.132$. This value is in agreement with the values calculated from previous works [31, 68, 50, 69] varying between 0.132 and 0.137 . From the spectra it can also be seen that three superharmonic frequencies are present, having significantly smaller amplitude; while Johnson and Patel [31] and Tomboulides and Orszag [68] also reported the presence of harmonics. The computed average values of $C_{D}$ and $C_{L}$ and their respective calculated amplitudes are presented in Table 2 and excellent agreement is found with the benchmark experimental and numerical results.

Figure 15 shows the three-dimensional vorticity structures, in the wake of the sphere, at an instant in time which corresponds maximum values of the lift, $C_{L}$, and drag, $C_{D}$, coefficients, respectively. These structures are identified by the $\lambda_{2}$ method [30] and colored with the velocity magnitude, normalized by the inlet velocity. Our simulations can capture correctly the hairpin vortices shed in the wake of the sphere and the presence of planar and non-axial symmetry. Interestingly, with only $3.2 \times 10^{6}$ cells, smooth coherent structures can be predicted in our simulation. This is attributed to the fact that the proposed grid refinement method allows constructing a locally uniform mesh in the wake of the sphere and avoids discontinuities across the interfaces with varying levels of refinement. Thus, no mesh stretching is needed and the second order accuracy of the solver is maintained.

\begin{tabular}{ccccccc}
\hline Authors & & $\overline{C_{D}}$ & $C_{D}^{\prime}\left(x 10^{-3}\right)$ & $\left|\overline{C_{L}}\right|$ & $C_{L}^{\prime}\left(x 10^{-2}\right)$ & $S t$ \\
\cline { 1 - 1 } Roos and Willmarth [54] & & 0.629 & - & - & - & - \\
Sakamoto and Haniu [55] & - & - & - & - & $0.148-0.165$ \\
Magnaudet et al. [40] & & 0.645 & - & - & - & - \\
Johnson and Patel [31] & & 0.656 & 3.5 & 0.069 & 1.6 & 0.137 \\
Tomboulides and Orszag [68] & 0.671 & 2.8 & - & - & 0.136 \\
Ploumhans et al. [50] & 0.683 & 2.5 & 0.061 & 1.4 & 0.135 \\
Vanella et al. [69] & 0.634 & 3.9 & - & - & 0.132 \\
Present 3-level refinement & 0.665 & 3.3 & 0.058 & 1.6 & 0.132 \\
\hline
\end{tabular}

Table 2: Comparison of the calculated values of the Strouhal number, $S t$, and the average values and oscillation amplitude of the drag, $\overline{C_{D}}, C_{D}^{\prime}$ and lateral $\overline{C_{L}}, C_{L}^{\prime}$ forces, respectively, against experimental measurements [54 55] and previous simulations [40 31 68 50 69], for the flow past a sphere at $R e=300$. Calculations performed on a 3-level refined grid consisted of $3.2 \times 10^{6}$ computational cells. 


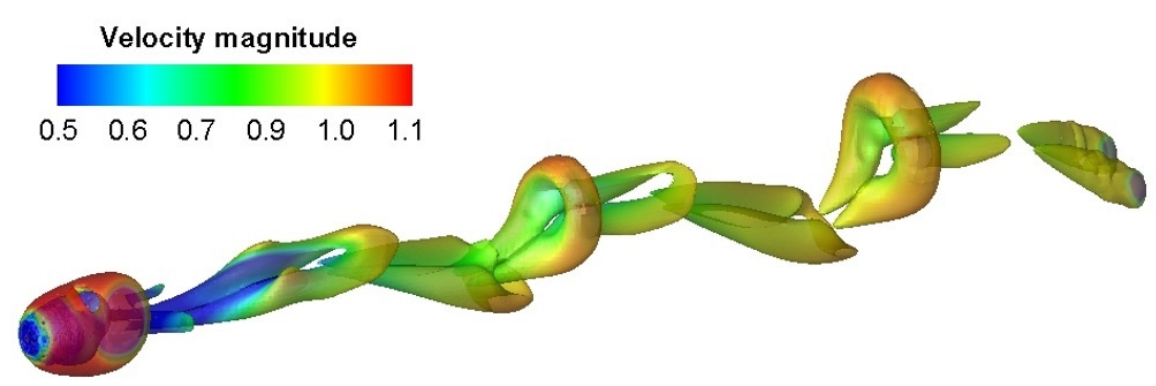

Fig. 15: Flow past a sphere at $R e=300$. Perspective view of the hairpin vortices identified by the $\lambda_{2}$ method [30] at an instant in time which corresponds maximum values of the lift, $C_{L}$, and drag, $C_{D}$, coefficients, respectively. The structures are colored with the velocity magnitude normalized by the bulk velocity. Calculations are performed on a 3-level refined grid consisted of $3.2 \times 10^{6}$ computational cells.

\subsection{Vortex shedding from a circular cylinder mounted between two endplates, $R e=100$ and 300}

An extensive body of literature, both experimental [71, 47] and computational [42, 66], has been devoted to understanding vortex shedding and wake dynamics past wall mounted circular cylinders. Various parameters including the Reynolds number, aspect ratio, blockage effect, end-conditions, wall roughness and inflow conditions effect the dynamics of vortex shedding. The wake characteristics past finite length cylinders are also influenced by the solid endplates that result in oblique shedding and may give rise to vortex dislocations.

We carry out herein direct numerical simulations of the flow past a wall mounted cylinder on locally refined meshes for $R e=100$ and 300, based on the cylinder diameter and inflow velocity. We consider a finite cylinder of aspect ratio $\lambda=L / D=16$ (where $L$ is the length and $D$ the diameter of the cylinder) located at $z=0$ and $12.5 D$ from the entrance of a rectangular computational domain extending between $-8 D \leq x \leq 8 D,-8 D \leq y \leq 8 D$ and $-12.5 D \leq z \leq 22.5 D$, with $(x)$ being the vertical, $(y)$ the spanwise and $(z)$ the streamwise directions, respectively. Uniform inflow profile is prescribed at the upstream boundary; the inlet velocity is $U_{\infty}$, free-slip conditions are specified at the vertical boundaries and no-slip conditions are applied at the spanwise boundaries and around the cylinder, which is treated as an immersed boundary and is represented by a triangular mesh of $3.2 \times 10^{5}$ elements. Even though Mittal [42] and Tang et al. [66] only considered one end plate in their simulations, using symmetry conditions at the mid-distance between the two end plates, and applied mixed boundary conditions at the plates (symmetry conditions from the inlet of the domain up to $4.5 \mathrm{D}$ upstream the cylinder and no-slip conditions on the rest of the plates) we consider herein the full domain with no-slip conditions applied on both end plates.

A primary Cartesian uniform grid $81 \times 41 \times 176$ is refined with special emphasis on increasing the resolution close to the solid walls. Numerical simulations are performed on six grids starting from the primary one and successively applying up to five levels of refinement. Concerning the refinement topology of the finest grid, refinement is applied within concentric cylinders, which cover the total spanwise length of the mounted cylinder, with the radii, ${ }^{k} R$, per level, $k$, of ${ }^{1} R=4.5 D$, ${ }^{2} R=1.5 D,{ }^{3} R=0.75 D,{ }^{4} R=0.6 D,{ }^{5} R=0.525 D$. Additionally, a rectangular region within $-4.5 D \leq x \leq 4.5 D$, $-8 D \leq y \leq 8 D$ and $0 \leq z \leq 20 D$ is also refined up to first level to achieve better resolution close to the spanwise endwalls and in the wake of the cylinder. For all our simulations, the selected time step corresponded to Courant number equal to $C F L=0.5$

Table 3 presents grid sensitivity analysis, at $R e=100$, and comparison of the calculated Strouhal number, $S t$, the mean drag coefficient, $\overline{C_{D}}$, and the amplitude of the lift coefficient oscillations, $C_{L}^{\prime}$, against previous experimental [71] and numerical [42, 66] studies. It is obvious that the solution without refinement and near-cylinder resolution of only 5 nodes per diameter fails to predict the flow physics and capture the dominant shedding frequency. Applying up to 5 levels of refinement around and in the wake of the cylinder resulted in a computational mesh of $10^{7}$ grid nodes and maximum wall distance $y^{+} \approx 3$. The calculated Strouhal number, $S t=0.154$, is in very good agreement with the prediction of Tang et al. [66], who found $S t=0.152$. This is attributed to the fact that for this value of Reynolds number, the dominant shedding frequency covers the whole span and the effect of the end walls on the frequency spectra is small. Thus, the different consideration of the spanwise boundary conditions does not affect the frequency calculation. This value compares well against the experimental measurements as well, considering not only the experimental uncertainties but the fact that both Norberg [47] and Williamson [71] found sudden drop in the dominant frequency from 0.161 to 0.141 for aspect ratio less than 30. Also, the time history and the power spectra of the lift and drag coefficients reveal that the flow is subjected to a single harmonic oscillation and the characteristic frequency of the drag variation is $f_{D}=0.310$.

In this study, the characteristic value of the average drag, $\overline{C_{D}}$, of the fully developed unsteady flow is found to be $\overline{C_{D}}=1.248$, in line with the computed values of [42, 66]. The calculated amplitude of the lift oscillations, $C_{L}^{\prime}$, is found to be $C_{L}^{\prime}=0.087$ which is in agreement with the reported value of [66] (Table 3). Overall our calculations achieve very good level of agreement compared to the work of [42, 66] assuming that in their simulations they did not consider no-slip conditions along the total length of the side-wall, where the cylinder was mounted, (but applied no-slip and symmetry conditions for $z>4.5 D$ and $z \leq 4.5 D$, respectively) and symmetry boundary conditions are imposed in the mid-span direction to facilitate the calculations. This also explains why the mean drag is slightly under-predicted compared to the corresponding works. However, the discrepancy between our results and the computations of Mittal [42], who found $C_{L}^{\prime}=0.047$, may be 
attributed to the different time increments of the computations; conclusion which is in line with works of [66].

\begin{tabular}{|c|c|c|c|c|c|c|c|}
\hline Source & $S t$ & $\overline{C_{D}}$ & $C_{L}^{\prime}$ & $\begin{array}{l}\text { Near wall } \\
\text { spacing/D }\end{array}$ & $\begin{array}{l}\text { Levels of } \\
\text { refinement }\end{array}$ & $\begin{array}{l}\text { Use of symmetry plane and } \\
\text { both symmetric and no-slip } \\
\text { conditions on the end plates }\end{array}$ & $\begin{array}{l}\text { Solution of the full domain } \\
\text { with no-slip conditions } \\
\text { applied on both end plates }\end{array}$ \\
\hline Tang et al. 66 & 0.152 & 1.325 & 0.075 & 0.006 & - & $\mathrm{x}$ & - \\
\hline Mittal [42] & 0.145 & 1.340 & 0.047 & 0.001 & - & $\mathrm{x}$ & - \\
\hline Williamson 71 & $\sim 0.141$ & - & - & - & - & - & - \\
\hline \multirow{6}{*}{ Present work } & 0.124 & 1.750 & 0.064 & 0.200 & - & - & $\mathrm{x}$ \\
\hline & 0.147 & 1.454 & 0.077 & 0.100 & 1 & - & $\mathrm{x}$ \\
\hline & 0.151 & 1.355 & 0.088 & 0.050 & 2 & - & $\mathrm{x}$ \\
\hline & 0.154 & 1.281 & 0.088 & 0.025 & 3 & - & $\mathrm{x}$ \\
\hline & 0.154 & 1.250 & 0.087 & 0.013 & 4 & - & $\mathrm{x}$ \\
\hline & 0.154 & 1.248 & 0.087 & 0.006 & 5 & - & $\mathrm{x}$ \\
\hline
\end{tabular}

Table 3: Comparison of the calculated values of the Strouhal number, $S t$, the average drag coefficient, $\overline{C_{D}}$, and the oscillation amplitude of the lateral forces $C_{L}^{\prime}$ against experimental measurements [71] and previous simulations [42 66], for the flow past a wall mounted cylinder of aspect ratio $\lambda=16$ at $R e=100$.

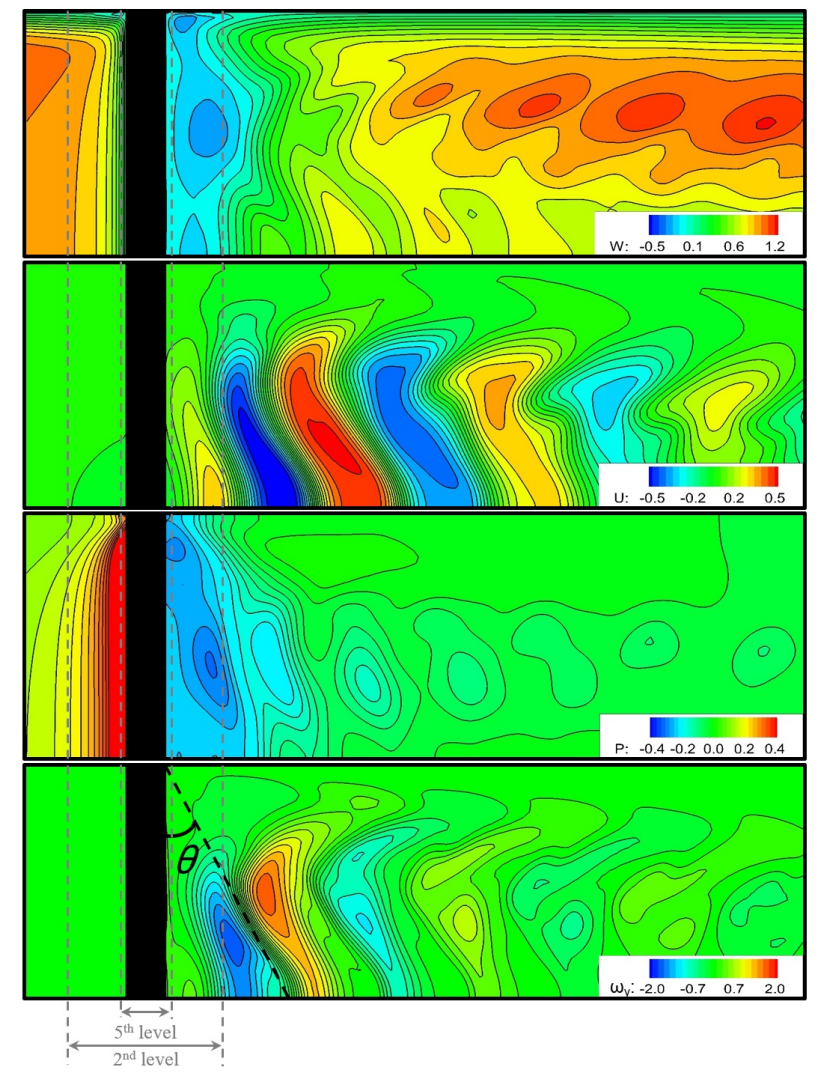

Fig. 16: Instantaneous half-slices at the plane $y=0$, showing the contours of the instantaneous velocity components, $W, U,($ along the $z$ and $x$ directions, respectively) pressure, $P$, and $y$-vorticity, $\omega_{y}$, for the fully developed unsteady flow past a wall mounted cylinder of aspect ratio $\lambda=16$ at $R e=100$. The plotted values are normalized by the bulk velocity, the diameter of the cylinder and the density of the fluid. Calculations performed on a 5-level refined grid consisted of $10^{7}$ computational cells.

To better investigate the shedding dynamics in the wake of the cylinder, we plot in Fig. 16 the instantaneous contours of the normalized velocity components, pressure and $y$-vorticity at a plane normal to the $x$ axis which is passing through the axis of the cylinder. It can be observed from this figure that the chevron-shaped are shed in an oblique but well organised manner. We can recognize the presence of the boundary layer close to the end-walls and the periodic oblique shedding accompanied by the existence of a single vortical cell covering the whole span. This conclusion agrees very well not only with previous numerical studies [42, 66] but also with the experimental observations of Williamson [71] who reported that for this range of Reynolds numbers and aspect ratio of the cylinder, a single chevron-shaped cell having a dominant frequency covers the whole span with periodic spectra. The shedding angle measured near the quarter span is found to be around $\theta=24.5^{\circ}$; this value agrees well with the value of $25^{\circ}$ calculated by Mittal [42]. Figure 16 also provides evidence for the smooth transition of all the flow properties across cells with different levels of refinement, owing to the hybrid staggered/non-staggered formulation on unstructured grids.

Two modes of three-dimensionality of the flow exist over a range of Reynolds numbers and their appearance is related to the discontinuity in the Strouhal-Reynolds number correlation. Above $R e=180$, the flow transitions from periodic to a disorganized quasi-periodic state resulting in Mode $A$ instability which is associated with discontinuous change in the wake formation. The primary wake vortical structures become unstable and large-scale finger-shaped vortex loops are generated [72, 56]. By further increasing the Reynolds number up to 230-260, a second discontinuity in the StrouhalReynolds function exists and the flow transitions to Mode B. Characteristic feature of this mode is the inception of finer 

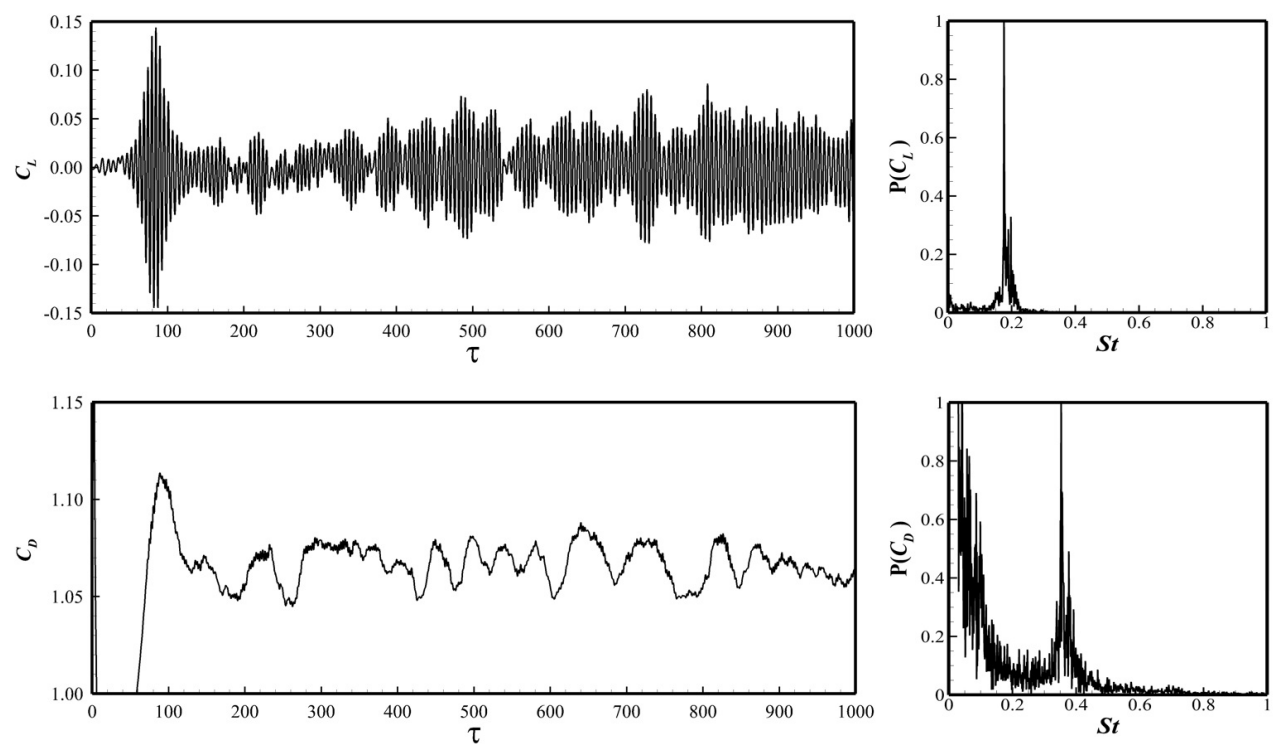

Fig. 17: Time history and single-sided spectrum of the lift, $C_{L}$, and drag, $C_{D}$, coefficients, respectively, for the flow past a wall mounted cylinder of aspect ratio $\lambda=16$, at $R e=300$. Calculations performed on a 5-level refined grid consisted of a total number of $10^{7}$ computational cells.

scale disorganized structures. The low frequency peak attributed to the Mode $A$ diminishes and a second peak at higher frequency appears, as a results of the shortwave length instabilities of Mode B. The parallel oblique shedding is inhibited by the transition from Mode $A$ to $B$ giving rise to the vortex dislocation phenomenon.

Herein, we are also investigating the flow past a wall mounted cylinder at $R e=300$. Our calculations are performed on the computational domain described above and the governing equations are discretized on the five-level refined mesh. Figure 17 shows the time history of the lift and drag coefficients and the corresponding power spectra. The calculated dominant lift frequency of $S t=0.18$ and the subdominant frequency of $f_{s u b}=0.20$ correspond to the characteristic frequencies of Modes $A$ and $B$, respectively, and are in good agreement with the results reported by Mittal [42] who found the above frequencies to be equal to 0.19 and 0.21 , respectively. The characteristic frequency was also found experimentally to have a value around 0.188 [47] for a case with larger aspect ratio equal to $L / D=20$. Also, the mean drag is found equal to $\overline{C_{D}}=1.07$ and agrees well with the value of 1.19 calculated by Mittal [42]. Any discrepancy with the previously reported simulations is attributed to the fact that we avoided the simplification of considering symmetry boundary conditions and we assumed that both the spanwise side-walls are subjected to no-slip boundary conditions along their total length, in contrast to [42, 66]. The vortex shedding mechanism is significantly influenced by the spanwise boundary conditions. In the previous calculations of Mittal [42] and Tang et al. [66] the limited extend of the no-slip condition resulted in the generation of non-smooth endwall boundary layer which effect the shedding mechanism and the corresponding frequency spectra. The low frequency irregularities of the lift fluctuation reveal dislocations (see Fig. 17), observation which is in line with previous simulations [42, 66], while those irregularities have also been experimentally found after analysing the velocity fluctuations in the wake of the cylinder [73]. Consequently, local mesh refinement does not have any negative effect on the spectrum of the induced forces on the cylinder and smooth transition of temporal scales is achieved.

Figure 18 presents the vortical structures in the wake of the cylinder, identified by the $\lambda_{2}$ method [30], coloured with the velocity magnitude, normalized by the bulk velocity, for the normalized instant in time $\tau=520\left(\tau=t \times U_{\infty} / D\right)$. For this Reynolds number, the flow enters a $3 D$ regime and perpendicular to the long spanwise structures, which constistute the Mode A instabilities, finger-shaped longitudinal vortices are also present (Mode B).

\subsection{Actuator line LES of wind turbine wake}

The actuator line (AL) parameterization of wind turbine blades is widely used in simulations of wind farms [36, 77, 59]. The AL approach eliminates the need for describing the details of the complex turbine geometry and has been shown to yield reasonably accurate descriptions of the turbine wake development [77]. Here we seek to illustrate that the numerical approach we have developed for discretizing and solving the incompressible Navier-Stokes equations on locally refined unstructured Cartesian grids can be implemented to carry out large-eddy simulation of wind turbine wakes with the AL parameterization. We apply the numerical method we have described herein to solve the spatially filter form of the NavierStokes equations with the subgrid scale eddy viscosity modeled using the standard Smagorinsky model [58].

In the AL approach the turbine blades are represented by lines rotating within the background unstructured three dimensional grid. Smooth distribution of forces along the actuator lines emulate the effect of the wind on the turbine rotor. The forces are function of the velocity field around the blades and the $2 \mathrm{D}$ airfoil characterists, including the drag $C_{D}$ and lift $C_{L}$ coefficients. The cross-sectional airfoil element at radius $r$ in the $(\theta, z)$ plane indicates that the local relative velocity $U_{\text {rel }}$ can be calculated from the velocity triangle as (Fig. 19a): 


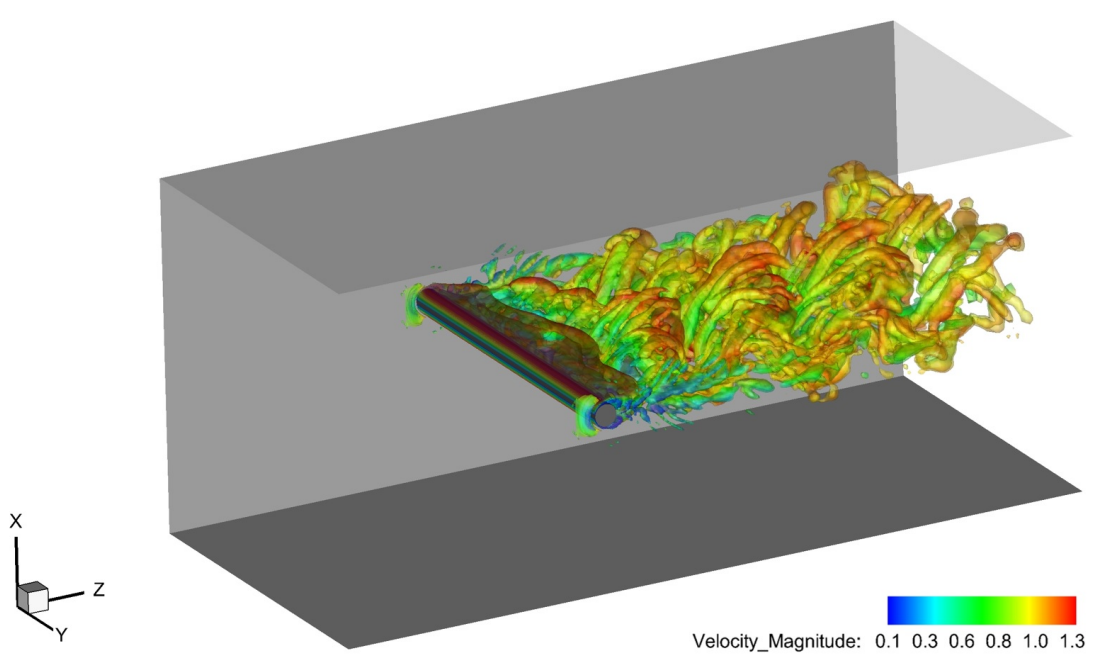

Fig. 18: Flow past a wall mounted cylinder of aspect ratio $\lambda=16$ at $R e=300$. Perspective view of the vortical structures identified by the $\lambda_{2}$ method [30] for the instant in time, normalized by the bulk velocity and the cylinder's diameter, $\tau=520$. The structures are colored with the velocity magnitude normalized by the bulk velocity. Calculations performed on a 5-level refined grid consisted of $10^{7}$ computational cells.

$$
U_{r e l}=\sqrt{U_{x}^{2}+\left(U_{\theta}-\omega r\right)^{2}}
$$

where $\omega$ is the angular velocity of the wind turbine rotor and $U_{\theta}$ and $U_{x}$ correspond to the tangential and axial velocity components, respectively. In this work, the velocity distribution along the actuator line grid points is obtained by interpolating from the cell centered Cartesian velocity components of the background unstructured grid (Fig. 19p). Smooth projection of the flow field can be ensured by employing a 3-point smoothed discrete delta function [78]. The local angle of attack $\alpha$ is a function of the local pitch angle $\gamma$ and the angle between the relative velocity and the rotor plane $\phi$ (Fig. 19. $)$ :

$$
\begin{gathered}
\alpha=\phi-\gamma \\
\phi=\operatorname{atan}\left(\frac{U_{x}}{U_{\theta}-\omega r}\right)
\end{gathered}
$$

Considering that the lift, $C_{L}$, and drag, $C_{D}$, coefficients are function of the angle of attack, $\alpha$, the lift $L$ and drag $D$ forces, per chord length $c$, can be calculated after having projected the velocity field along the actuator line grid points:

$$
\begin{aligned}
& L=c \frac{1}{2} C_{L}(\alpha) \rho U_{r e l}^{2} \\
& D=c \frac{1}{2} C_{D}(\alpha) \rho U_{r e l}^{2}
\end{aligned}
$$

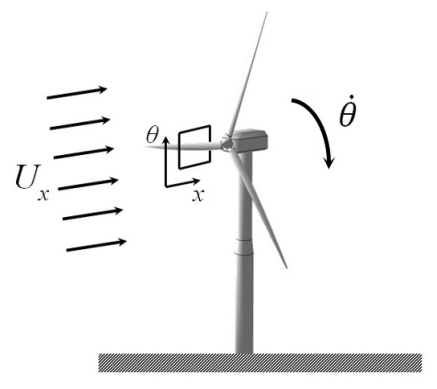

(a)

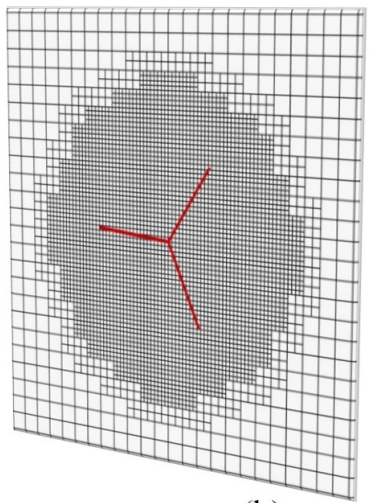

(b)

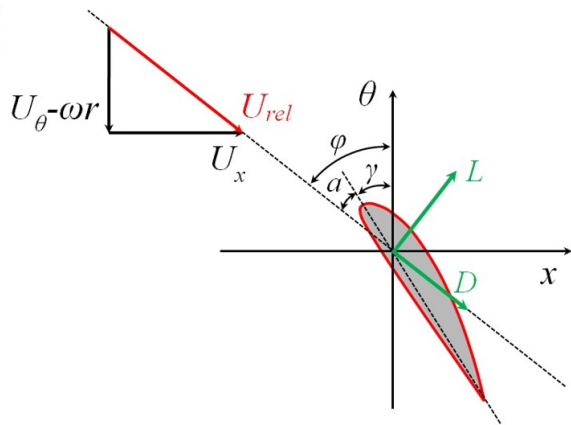

(c)

Fig. 19: Schematic representation of the actuator line model. (a) Sketch of a turbine and a two dimensional section of the blade. (b) Actuator lines immersed within an unstructured Cartesian mesh used to represent the blades of a turbine. (c) Velocity and force analysis with respect to the axial and tangential coordinates. 

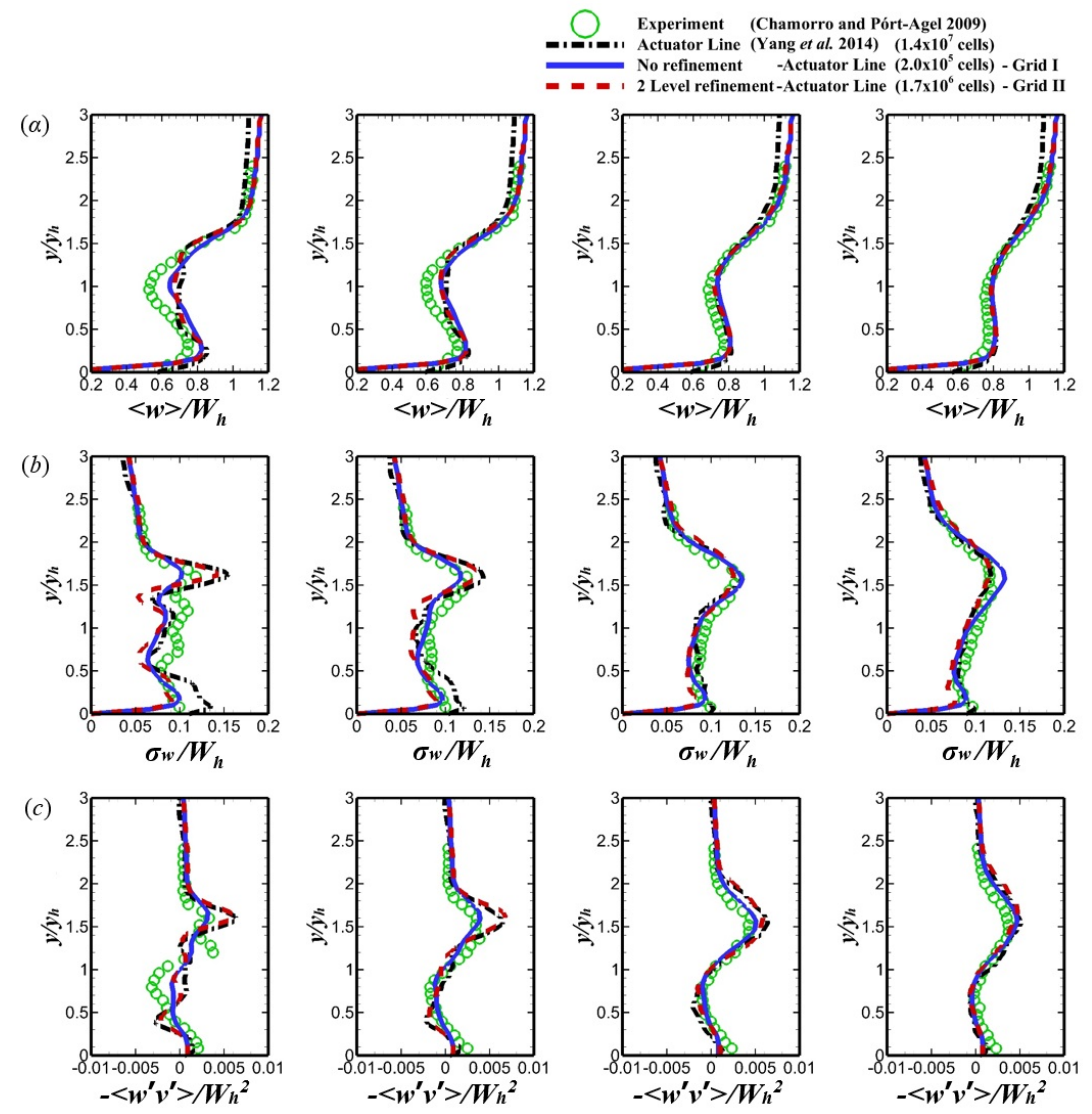

$z / D=2$

$z / D=3$

$z / D=5$

$z / D=7$

Fig. 20: Comparison of the vertical profiles of mean flow quantities, at different locations downstream the wind turbine [16], calculated on a uniform Cartesian grid $51 \times 31 \times 136$ without refinement (grid I) and on a 2-level refined grid adapted around and in the wake of the wind turbine's rotor (grid II), against previous experimental measurements [16] and numerical simulations [77]. (a) Streamwise velocity, (b) streamwise turbulence intensity, and (c) Reynolds shear stress component. Here $\sigma_{w}$ is the streamwise turbulence intensity, $v$ and $w$ are the instantaneous velocities in the $y$, and $z$ directions, respectively, and $W_{h}$ is incoming velocity at the hub height. The prime symbol denotes fluctuating quantities and $<\ldots>$ indicates the time averaging operation.

We carry out LES over a stand-alone miniature wind turbine and the numerical setup corresponds to the experimental work performed by Chamorro and Pórte-Agel [16]. The experimental work investigated the flow field over a miniature turbine consisted of a three-blade GWS/EP- $6030 \times 3$ rotor attached to a small DC generator with turbine diameter $D=0.15 \mathrm{~m}$ and hub height $h=5 D / 6$. The Tip Speed Ratio (TSR) of the turbine is $\lambda=4.1$ and the Reynolds number, normalized by the incoming velocity, $W_{h}$, at the hub height, $y_{h}$, and the rotor diameter, $D$, is $R e=4.2 \times 10^{4}$. The same problem was also studied numerically by Wu and Pórte-Agel [76] who treated the blades as a flat plate and by Yang et al. [77] who represented the blades by a NACA0012 [57]. In the present simulations, we followed the latter more realistic approach; further details can be found in [3].

The governing equations are discretized on a computational domain sized $5 D, 3 D$ and $20 D$ in the spanwise $(x)$, vertical $(y)$ and streamwise $(z)$ directions, respectively. Two simulations are carried out, the first is performed on a uniform Cartesian grid (grid I) with $N_{x}=51, N_{y}=31$ and $N_{z}=136\left(\sim 2 \times 10^{5}\right.$ cells) $\left(N_{x}, N_{y}\right.$ and $N_{z}$ are the number of grid nodes in $x, y$ and $z$ directions, respectively) and the second is performed on a locally refined mesh (grid II) which is generated after remeshing the primary uniform Cartesian grid (grid I) in an ellipsoidal manner around the rotor of the turbine by applying two levels of refinement. The refined region extends from $2 D$ upstream to $14 D$ downstream the rotor of the turbine. Hence, the refined grid consisted of $1.7 \times 10^{6}$ cells and the equivalent uniform Cartesian grid having spacing equal to the finest level of grid II would consist of $1.3 \times 10^{7}$ cells. The resulting resolution allowed representation of the rotor with 10 and 40 nodes per diameter, on grids I and II, respectively. Pre-computed fully developed turbulent flow is used to generate inflow conditions, free slip conditions are applied at the top and spanwise boundaries and a wall model is utilized at the bottom bed [70]. The inlet profile is the same in all our simulations and the time step is equal to $\tau=0.001 \mathrm{D} / \mathrm{W}_{h}$.

The distribution of the time-averaged streamwise velocity, $\langle w\rangle$, turbulence intensity, $\sigma_{w}$ and the Reynolds shear stress component $-\left\langle w^{\prime} v^{\prime}\right\rangle$, normalized by the incoming hub height velocity, $W_{h}$, at several locations downstream of the turbine, is shown in Fig. 20, Our results are compared against the experimental measurements of [16] and previous simulations of [77]. The spanwise resolution around the rotor achieved on the refined grid II is equivalent to the spatial discretization of the numerical investigation by Yang et al. [77] who solved the same problem by using a $481 \times 241 \times 121$ structured stretched grid to discretize a $30 D \times 12 D \times 3 D$ domain. The simulated time-averaged streamwise velocity profiles (Fig. 201) are in good agreement with the experimental measurements, mainly in the far-wake regions with $z / D>5$. The 
velocity over prediction in the near wake region at the hub height, is attributed to the fact that both the nacelle and the tower are not modelled in the AL approach. This is a well known shortcoming of the actuator line parameterization as it is inherently insufficient for accurate near wake calculations for $z / D<2$ [77]. Overall, good prediction of the distribution of the streamwise turbulence intensity is achieved on the refined grid (Fig. 20p). The over prediction of the turbulent intensity at the top tip of the turbine further downstream at $z / D=7$ is eliminated on the refined mesh and the simulated trends are in good agreement with [16] and [77]. Concerning the distribution of the Reynolds shear stress component $-\left\langle w^{\prime} v^{\prime}\right\rangle$ (Fig. $20 \mathrm{c}$ ), our results are found to be in line with the previous studies. However, the actuator line parameterization in any case fails to provide reliable results in the near wake region of $z / D \leq 2$. This can explain why the calculations on the refined mesh agree very well with the calculations of [77] who solved the same problem on a structured grid of $1.4 \times 10^{7}$ grid nodes. The AL is insufficient to provide accurate calculations close to the wake of the turbine due to the fact that the nacelle is not modelled and instead the blade geometry is extended to the center of the rotor. Also, the representation of the blades with lines of zero-thickness imply that the actual thickness of the blades is not considered in this model and, as a results, additional uncertainty is introduced into the calculations.

Qualitatively, the velocity deficit in the near wake is related to the energy extracted by the turbine; an effect which influences the flow field even in the far wake. The rotation of the turbine generates large shear stresses which originate from near the rotor tip (Fig. 20,) which agrees with the experimental observations of [76]. The rich dynamics resolved on the unstructured grid are shown in Fig. 21 where the instantaneous streamwise velocity contours are displayed.

\subsection{Geometry resolving LES of hydrokinetic turbine}

In this last simulated test case we seek to illustrate the potential of our numerical method to carry out LES of hydrokinetic turbines while resolving via the Immersed Boundary method (rather than parameterizing with the AL method) all geometrical details of the turbine, including the blades, nacelle and the tower. We choose the experiments of Chamorro et al. [15] which involve a very complex hydrokinetic turbine geometry and has also been studied numerically using the CURVIB method on structured Cartesian grids by Kang et al. [33].

The model turbine with diameter $D=0.5 \mathrm{~m}$ was placed in the main channel of the St. Anthony Falls Laboratory (SAFL) of the University of Minnesota. This channel is $2.75 \mathrm{~m}$ wide and $85 \mathrm{~m}$ long and the mean flow depth of the water $(H)$ during the experiments was $1.15 \mathrm{~m}$. The turbine was located in the center of the channel so that the flow was fully-developed before it encounters the turbine. The bulk mean velocity in the channel was $U=0.4 \mathrm{~m} / \mathrm{s}$. The Reynolds number based on bulk mean velocity and turbine diameter was $R e=1.7 \times 10^{5}$. The turbine was rotating with an angular speed of $\omega=9.43 \mathrm{rad} / \mathrm{s}$ about its axis, resulting in a TSR of $\lambda=5.89$. More details of the experiments can be found in [15].

Similar to [33], a subsection of the main channel $9 D$ long $(z), 5 D$ wide $(x)$ and $2.3 D$ deep $(y)$ is chosen as computational domain. Inside the computational domain, the rotating center of the turbine is placed at $(0,0.8 D, 0)$ and the base of the pylon is at $(0,0,-0.3 D)$. The inlet is fed with a fully developed channel flow pre-computed from a separate LES of periodic channel flow. The channel of this LES had same cross section as the computational domain for turbine simulation. The two channel side walls and the bottom bed were assumed to be hydrodynamically smooth. Wall-modeling approach of Wang and Moin [70] was used to reconstruct velocity boundary conditions at all turbine surface and channel walls; the details of implementing the wall model in the context of the Immersed Boundary method can be found in [12]. At the free surface of the domain, zero-flux and free-slip boundary conditions are employed.

The primary uniform Cartesian grid for this case has $N_{x}=126, N_{y}=58$ and $N_{z}=451$ grid nodes $\left(N_{x}, N_{y}\right.$ and $N_{z}$ are the number of grid nodes in $x, y$ and $z$, respectively) resulting in a resolution of $25 \times 25 \times 50$ cells per rotor diameter, along $x, y$ and $z$ directions, respectively (level zero). This is locally refined near the turbine rotor by applying two levels of refinement around and in wake of the turbine, as shown in Fig. 22 The resulted refined grid had maximum resolution of $100 \times 100 \times 200$ cells per rotor diameter, along $x, y$ and $z$ directions, in the immediate vicinity of the turbine and a total

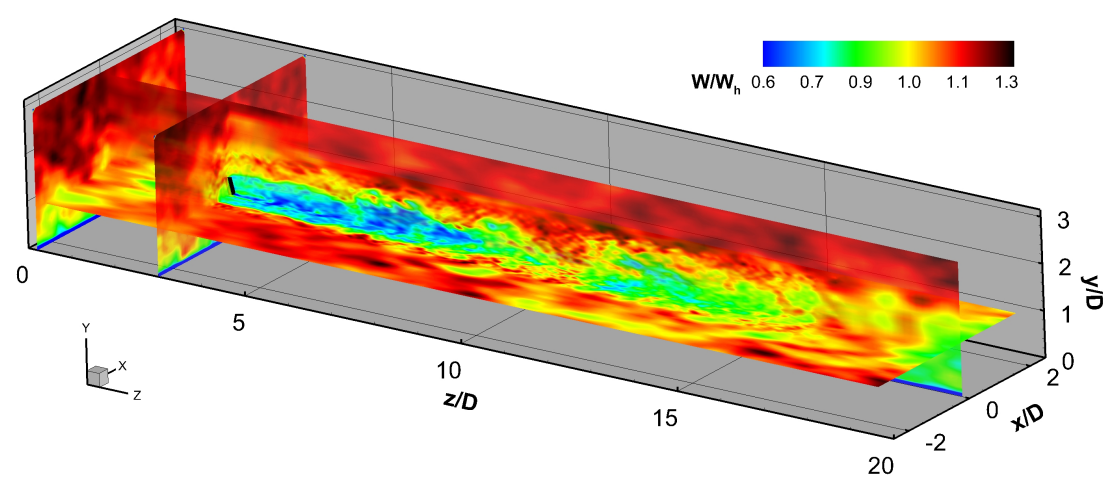

Fig. 21: Contours of the actuator line computed instantaneous streamwise velocity, $w$, normalized by the incoming velocity at the hub height, $W_{h}$, on the planes across the hub and parallel to the rotor, for the flow around a miniature wind turbine [16] with tip speed ratio $\lambda=4.1$ at $R e=4.2 \times 10^{4}$. Calculations are performed on a 2-level refined mesh and the inlet is fed with a fully developed channel flow pre-computed from a separate LES. 
of $8 \times 10^{7}$ cells. The simulations were first run for 25 revolutions until the total kinetic energy of the domain reached a quasi-steady state. Subsequently, the results were time averaged for another 55 rotor revolutions.

Figure 23 presents the instantaneous contours of the streamwise velocity on the $x=0$ and $y / D=0.8$ planes across the hub. It is evident that the energetic, slowly precessing low streamwise velocity region originates in the immediate vicinity of the nacelle, as was also identified by Kang et al. [33] who extensively studied its importance on the far wake development. The simulations also capture the onset of far wake meandering that manifests itself as large-amplitude, low-frequency lateral fluctuations starting at approximately 3D downstream of the rotor. These findings are in excellent agreement with the findings of Kang et al. [33] regarding the rich coherent dynamics of the turbine wake and the importance of accurately resolving the details of the turbine nacelle for capturing such dynamics (Fig. 23 ).
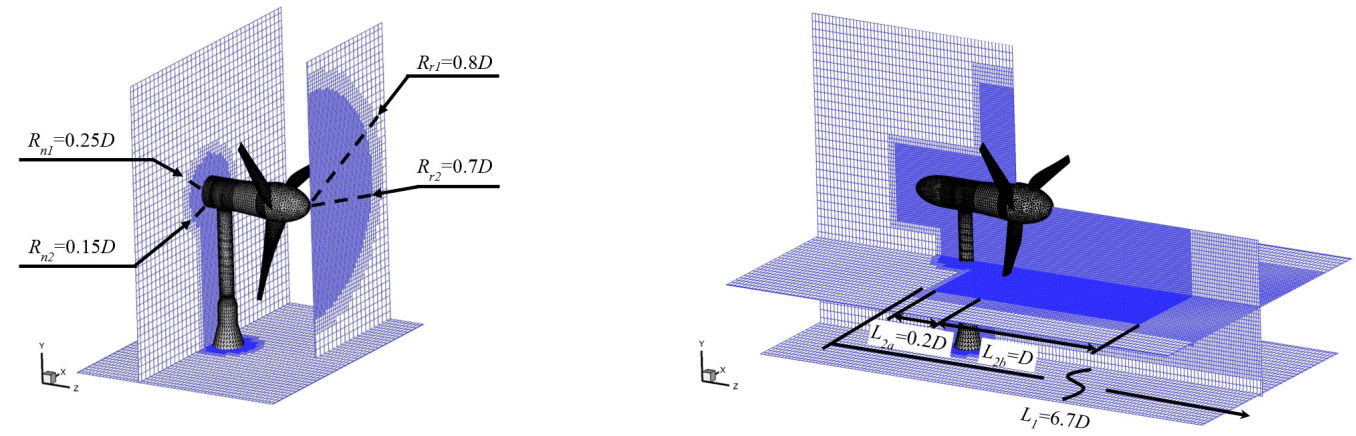

Fig. 22: 2-level local mesh refinement adapted around and in the wake of a hydrokinetic turbine [15]. A primary uniform Cartesian grid $126 \times 58 \times 451$ is refined resulting in a maximum resolution of $100 \times 100 \times 200$ cells per rotor diameter in the vicinity of the turbine and $8 \times 10^{6}$ computational cells. The hydrokinetic turbine is discretized using a triangular mesh of $1.1 \times 10^{4}$ elements.

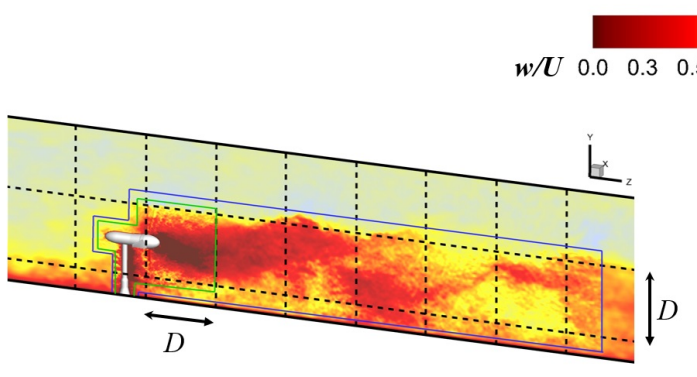

$(\alpha)$

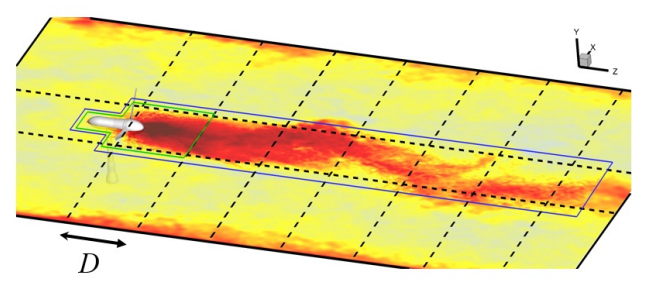

(b)

Fig. 23: Contours of the instantaneous streamwise velocity $w$, normalized by the bulk velocity $U$, on planes across the hub: (a) $x=0$; (b) $y=0.8 D$, for the geometry resolving LES around a hydrokinetic turbine [15] with tip speed ratio $\lambda=5.89$ at $R e=1.7 \times 10^{5}$. Calculations are performed on a 2 -level refined mesh and the inlet is fed with a fully developed channel flow pre-computed from a seperate LES. The blue and green solid lines indicate the regions where the $1^{\text {st }}$ and $2^{\text {nd }}$ levels of refinement applied, respectively.

In Fig. 24 , the vertical profiles of the mean streamwise velocity are compared against experimental measurements [15] and simulations of the same case using the AL approach and previous geometry resolving LES [33]. The 2-level turbine resolving LES calculations agree well with the experimental measurements of [15]. Discrepancies between our results and the measurements may be attributed to the fact that the pre-computed LES of fully developed channel flow fed at the inlet performed on the coarse primary grid to avoid any interpolation at the inlet of the domain. Also, further grid refinement is expected to improve velocity deficit prediction. Comparison with the AL calculations of [33] reveal that the over-prediction of the streamwise velocity in the near wake region of the turbine which is inherent in AL models [77, 33] is avoided by resolving the details of the hub and nacelle geometry. Our results also compare also well with the geometry resolving simulations of [33] who solved the same problem on a structured grid using the CURVIB solver [12]. It is remarkable that with our method we achieve the same level of agreement by using 20 times smaller number of computational cells.

The computed vertical profiles of the turbulence kinetic energy (TKE) are shown in Fig. 24p. It can be observed that the pockets of high TKE that emanate from the nacelle and the tip vortices merge together and the TKE at upper tip height suddenly starts to increase when $\mathrm{z}>3 \mathrm{D}$, in agreement with the study of [33]. The over prediction of the computed TKE levels in the near weak region around the hub height may indicate that higher grid resolution is needed. However, in the far wake $(\mathrm{z}>3 \mathrm{D})$ our calculations are in very good agreement with the experimental measurements of [15], while the geometry resolving calculations of [33], who used very fine stretched Cartesian grid, under-predict the levels of turbulence kinetic energy. It is demonstrated that with our method the induced turbulence mixing can be accurately resolved on locally refined grids. Even though the AL simulations predict correctly the TKE levels on a coarse structured grid [33], the inherent weaknesses of the actuator line parameterization concerning the accuracy of near wake calculations and the non-grid independent solution of such methods have been discussed in [77, 33].

Detailed turbine resolving calculations are currently under way and results concerning near- and far-wake mean velocity and turbulence statistics on adaptively refined grids will be reported in a future communication. Turbine resolving 
$(\alpha)$

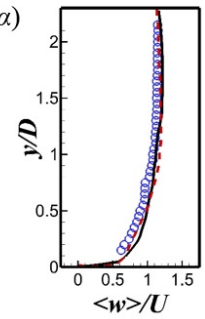

(b)

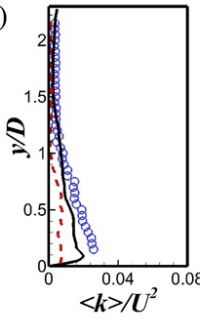

$z / D=-1$
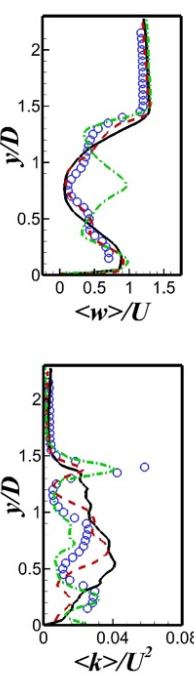

$z / D=1$
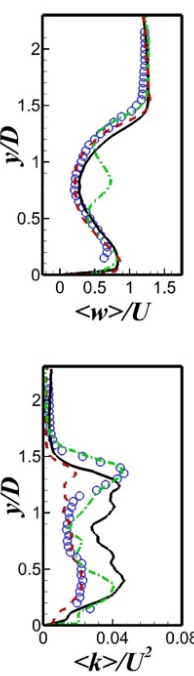

$\mathrm{z} / \mathrm{D}=2$
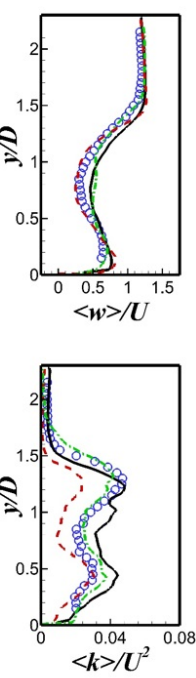

$z / D=3$

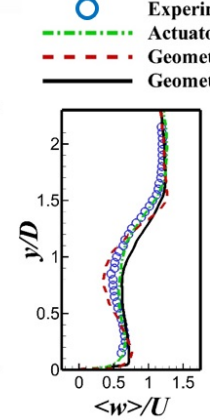
Actuator Lin

(Chamorro et al. 2013) (Kang et al. 2014) (3.9x107 cells) (Kang et al. 2014) $\left(1.6 \times 10^{8}\right.$ cells $)$
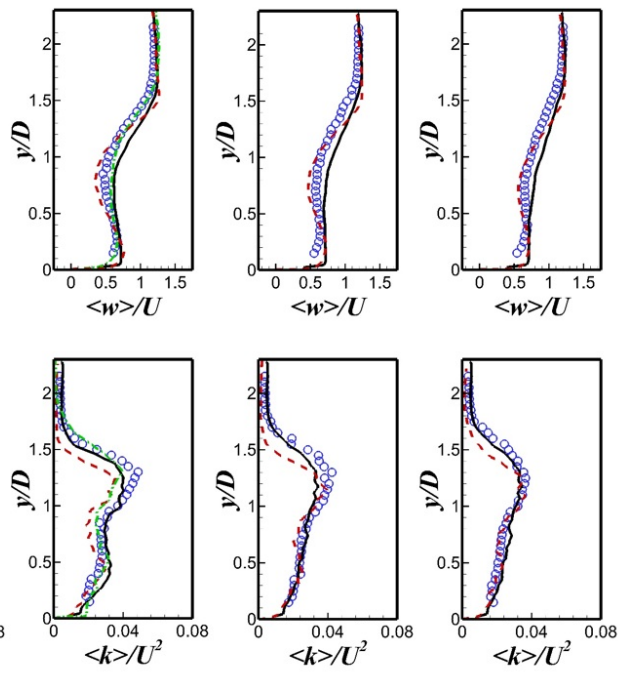

$z / D=4$

$z / D=5$

$z / D=6$

Fig. 24: Comparison of the vertical profiles of mean flow quantities, at different locations downstream the hydrokinetic turbine [15], of geometry resolving LES computed on a 2-level refined grid, against previous calculations [33] and experimental measurements [15]. (a) Streamwise velocity, (b) turbulence kinetic energy. Here $w$ and $k$ are the instantaneous streamwise velocity and the turbulence kinetic energy, respectively, and $<\ldots>$ indicates the time averaging operation.

calculations on locally refined meshes will also enable accurate multi-turbine simulations.

\section{Summary and conclusion}

We have developed a novel method for discretizing and solving the unsteady incompressible Navier-Stokes equations on unstructured Cartesian grids. Our work is inspired by the method of [26], where the hybrid staggered/non-staggered grid layout was introduced for solving the Navier-Stokes on structured Cartesian grids. Our method, however, constitutes a major algorithmic advance as it provides a powerful and general computational approach for solving the governing equations on locally refined Cartesian grids while satisfying the discrete continuity equation to machine zero without the flux mismatch and pressure discontinuities issues that plague other local refinement approaches. The numerical method enables adaptive mesh refinement in the vicinity of arbitrarily complex immersed boundaries and is used to augment the accuracy of the sharp interface immersed boundary method of [12] without requiring excessive computational resources. The discrete equations are solved using an unstructured monolithic approach employing a matrix-free Krylov method for the momentum equations and the Krylov-subspace methods for the Pressure Poisson equation. Such monolithic solution method on an unstructured Cartesian grid with local refinement is advantageous for parallel computing implementation because: a) nonoverlapping information is used, b) the parallel domain decomposition procedure is straightforward and c) load balance can be achieved after calling a conventional repartitioning function. The numerical method is capable for carrying out direct numerical simulations (DNS) and large-eddy simulations (LES) of flows involving arbitrarily complex geometries.

Extensive grid sensitivity analysis of a Poisson-like problem demonstrated the second-order accuracy of the discretization method. The flow around a sphere for Reynolds numbers range of $20 \leq R e \leq 300$ demonstrated the ability of our method to accurately simulate steady and unsteady regimes and capture the underlying physics of the flow showing good level of agreement with numerical and experimental studies. The same test case was also used to demonstrate significant efficiency gains as compared to calculations on structured grids. The chevron-shaped oblique shedding from a finite circular cylinder mounted between two end walls was also studied and the potential of our method for simulating complex vortex shedding phenomena, such as the appearance of Modes $A$ and $B$ shedding at $R e=300$ was illustrated. The above calculations also revealed the smooth transition of temporal and spatial scales, across different grid resolution interfaces, which is achieved on complex irregular flows. Finally the potential of our method for carrying out hydro- or wind-turbine LES using both the AL approach as well as the geometry-resolving approach was illustrated. In all the cases we were able to predict the prevailing flow physics on locally refined grids by using a small fraction of the resources needed in previously reported simulations performed on structured meshes. In our future work we will focus on extending the method to simulate large wind farms interacting with atmospheric turbulence. Our method is especially promising as a tool for carrying out simulations at such scale by resolving, rather than parameterizing, the wind turbine geometry details. We note that while our emphasis is on DNS and LES of complex flows, our method can also efficiently incorporate the Reynolds-averaged Navier-Stokes (RANS) equations to simulate turbulent flows. Given that RANS methods are tolerant to using grids with 
cells of very high aspect ratios, the RANS module in our solver could facilitate calculations on locally refined meshes with highly non-uniform cells. This will enable us to perform low cost simulations of practical engineering turbulent flows.

\section{Acknowledgements}

This work has been supported by the US Department of Energy (DE-EE 0005482), the US National Science Foundation (CBET-1341062, CBET-1622314) and the University of Minnesota Initiative for Renewable Energy and the Environment. Computational resources were provided by the Minnesota Supercomputing Institute.

\section{Appendix A. Calculation of weighting factors of the hanging nodes by using bi-quadratic quadrilateral interpolation}

In this work smooth isotropic refinement of the Cartesian grid is chosen and the difference of the levels of refinement between adjacent cells cannot be greater than one. Essentially, taking advantage of the cells' topology, the interpolated values at the hanging nodes can be expressed as a function of the stencil's values and weighting factors. It has already been stated that the hanging nodes are used whenever a grid cell is surrounded by other cells with different levels of refinement. In such a case, a two-dimensional $3 \times 3$ patch forms the two dimensional reconstruction stencil (Fig. 25). The latter stencil consists of a $3 \times 3$ uniform subgrid $(3 \Delta \xi, 3 \Delta \eta)$ with $\Delta \xi, \Delta \eta$ being the dimensions of the current coarse cell along the plane were reconstruction is applied. Obviously, the $3 \times 3$ values of an arbitrary variable $\phi$ of the stencil coincide with the cell centered values of the computational grid if the surrounding cells have same level of refinement as the reference cell. Otherwise, the nodes of the stencil may be located at the center of an isotropically split cell and an inverse distance function is utilized to calculate a value at the corresponding location of the stencil. The $3 \times 32 \mathrm{D}$ Lagrange interpolation, which practically results in a quadrilateral bi-quadratic interpolation, enables the calculation of the 9 weighting factors $L_{i}(\xi) L_{j}(\eta)$, $i, j=0,2$ (see Eq. (3) ) unless nodes of computational stencil are lying on the boundary of the computational domain. The equivalent polynomial formulation can be used in order to calculate the weighting factors in the general case that parts of the computational stencil may be lying along the boundaries governed by Dirichlet or Neumann conditions.

Assuming that $f_{i j}=f\left(\xi_{i}, \eta_{j}\right)$, the 2D Langrange interpolation on a $3 \times 3$ stencil can be described from Eq. 6 as:

$$
\phi(\xi, \eta)=\sum_{i=0}^{2} \sum_{j=0}^{2} w_{i j}(\xi, \eta) f_{i j}=\sum_{k=0}^{8} \widetilde{w}_{k} \widetilde{f}_{k}
$$

However, the 9-point quadrilateral bi-quadratic interpolation has the following form:

$$
\phi(\xi, \eta)=\left[\begin{array}{lllllllll}
1 & \xi & \eta & \xi \eta & \xi^{2} \eta & \xi \eta^{2} & \xi^{2} & \eta^{2} & \xi^{2} \eta^{2}
\end{array}\right]\left[\begin{array}{c}
c_{0} \\
c_{1} \\
c_{2} \\
c_{3} \\
c_{4} \\
c_{5} \\
c_{6} \\
c_{7} \\
c_{8}
\end{array}\right]
$$

with $c_{i},(i=0,8)$ being unknown coefficients which depend on the arrangement of the stencil and the interpolation position $(\xi, \eta)$.

Assuming now that reconstruction is performed within the $(3 \Delta \xi, 3 \Delta \eta)$ stencil (see Fig. 25 (a)), the $\widetilde{f_{i}}, i=0,8$ known values satisfy the biquadratic interpolation with: $\widetilde{f}_{0}=\phi(0,0), \widetilde{f}_{1}=\phi(\Delta \xi, 0), \widetilde{f}_{2}=\phi(2 \Delta \xi, 0), \widetilde{f}_{3}=\phi(0, \Delta \eta), \widetilde{f}_{4}=\phi(\Delta \xi, \Delta \eta)$, 
$\widetilde{f}_{5}=\phi(2 \Delta \xi, \Delta \eta), \widetilde{f}_{6}=\phi(0,2 \Delta \eta), \widetilde{f}_{7}=\phi(\Delta \xi, 2 \Delta \eta), \widetilde{f}_{8}=\phi(2 \Delta \xi, 2 \Delta \eta)$ and from Eqs. 46 , 47 the weighting factor vector $\widetilde{\mathbf{w}}$ may be calculated:

$$
\widetilde{\mathbf{w}}(\xi, \eta)=\left[\begin{array}{c}
1 \\
\frac{\xi}{\Delta \xi} \\
\frac{\eta}{\Delta \eta} \\
\frac{\xi \eta}{\Delta \xi \Delta \eta} \\
\frac{\xi^{2} \eta}{\Delta \xi^{2} \lambda \eta} \\
\frac{\xi \eta^{2}}{\Delta \xi \Delta \eta^{2}} \\
\frac{\xi^{2}}{\Delta \xi^{2}} \\
\frac{\eta^{2}}{\Delta \eta^{2}} \\
\frac{\xi^{2} \eta^{2}}{\Delta \xi^{2} \Delta \eta^{2}}
\end{array}\right]\left[\begin{array}{ccccccccc}
1 & 0 & 0 & 0 & 0 & 0 & 0 & 0 & 0 \\
-\frac{3}{2} & 2 & -\frac{1}{2} & 0 & 0 & 0 & 0 & 0 & 0 \\
-\frac{3}{2} & 0 & 0 & 2 & 0 & 0 & -\frac{1}{2} & 0 & 0 \\
\frac{9}{4} & -3 & \frac{3}{4} & -3 & 4 & -1 & \frac{3}{4} & -1 & \frac{1}{4} \\
-\frac{3}{4} & \frac{3}{2} & -\frac{3}{4} & 1 & -2 & 1 & -\frac{1}{4} & \frac{1}{2} & -\frac{1}{4} \\
-\frac{3}{4} & 1 & -\frac{1}{4} & \frac{3}{2} & -2 & \frac{1}{2} & -\frac{3}{4} & 1 & -\frac{1}{4} \\
\frac{1}{2} & -1 & \frac{1}{2} & 0 & 0 & 0 & 0 & 0 & 0 \\
\frac{1}{2} & 0 & 0 & -1 & 0 & 0 & -\frac{1}{2} & 0 & 0 \\
\frac{1}{4} & -\frac{1}{2} & \frac{1}{4} & -\frac{1}{2} & 1 & -\frac{1}{2} & \frac{1}{4} & -\frac{1}{2} & \frac{1}{4}
\end{array}\right]
$$

Having defined $\widetilde{\mathbf{w}}$, the desired value of any dependent variable $\phi$ at an arbitrary position $(\xi, \eta)$ of the two-dimensional stencil which is not adjacent to walls of the domain, can be calculated from Eq. (46). In our calculations, polynomial reconstruction is required at the following locations of the stencil: $\left(\frac{3}{4} \Delta \xi, \frac{3}{4} \Delta \eta\right),\left(\frac{3}{4} \Delta \xi, \frac{5}{4} \Delta \eta\right),\left(\frac{5}{4} \Delta \xi, \frac{5}{4} \Delta \eta\right)$ or $\left(\frac{5}{4} \Delta \xi, \frac{3}{4} \Delta \eta\right)$ where the hanging nodes are located. Thus, the values of those coefficients are explicitly calculated and obtain unique values on isotropically refined grids. Following similar reasoning, weighting factors can be calculated in a straightforward manner if data points from the stencils are governed by specific boundary conditions. For instance, if nodes are governed by Neumman or Dirichlet boundary conditions, the zero gradient condition or specific values can be enforced at the data points. Table 4 summarizes the calculated weighing factors, corresponding to Eq. (45), which are function of the interpolation position and possible boundary conditions around the computational stencil (Fig. 25). Expectedly, the summation of the weighting factors is always equal to one. It should be noted that for calculations on hanging nodes which belong to corner cells, the contribution of the $\Delta \xi^{2} \Delta \eta^{2}$ in Eq. 477 is neglected. The pre-calculation of the weighting factors is important because the bi-quadratic polynomial reconstruction around the coarse/fine cells can be performed with low computational cost by weighting the contribution of the values of the cells around the hanging nodes.

\begin{tabular}{|c|c|c|c|c|c|c|c|c|c|c|c|}
\hline \multirow{2}{*}{$\frac{\text { Position }}{(\xi / \Delta \xi, \eta / \Delta \eta)}$} & \multicolumn{9}{|c|}{ Weighting factors $\left(\mathrm{x} \frac{1}{1024}\right)$} & \multirow[t]{2}{*}{ Boundary condition } & \multirow[t]{2}{*}{ Boundary nodes } \\
\hline & $\widetilde{w}_{0}$ & $\widetilde{w}_{1}$ & $\widetilde{w}_{2}$ & $\widetilde{w}_{3}$ & $\widetilde{w}_{4}$ & $\widetilde{w}_{5}$ & $\widetilde{w}_{6}$ & $\widetilde{w}_{7}$ & $\overline{w_{8}}$ & & \\
\hline$\left(\frac{3}{4}, \frac{3}{4}\right)$ & 25 & 150 & -15 & 150 & 900 & -90 & -15 & -90 & $\overline{9}$ & - & - \\
\hline$\left(\frac{3}{4}, \frac{3}{4}\right)$ & 25 & 150 & -15 & 135 & 810 & -81 & - & - & - & Neumann & $6,7,8$ \\
\hline$\left(\frac{3}{4}, \frac{5}{4}\right)$ & -15 & -90 & 9 & 175 & 1050 & -105 & - & - & - & Neumann & $6,7,8$ \\
\hline$\left(\frac{3}{4}, \frac{3}{4}\right)$ & 16 & 144 & - & 144 & 720 & - & - & - & - & Neumann & $2,5,6,7$ \\
\hline$\left(\frac{5}{4}, \frac{5}{4}\right)$ & -16 & -80 & - & -80 & 1200 & - & - & - & - & Neumann & $2,5,6,7$ \\
\hline$\left(\frac{3}{4}, \frac{5}{4}\right)$ & 0 & -96 & - & 160 & 960 & - & - & - & - & Neumann & $2,5,6,7$ \\
\hline$\left(\frac{3}{4}, \frac{3}{4}\right)$ & 20 & 120 & -12 & 180 & 1080 & -108 & -40 & -240 & $\overline{24}$ & Dirichlet & $6,7,8$ \\
\hline$\left(\frac{3}{4}, \frac{5}{4}\right)$ & $-20 / 3$ & -40 & 4 & 100 & 600 & -60 & $200 / 3$ & 400 & -40 & Dirichlet & $6,7,8$ \\
\hline$\left(\frac{3}{4}, \frac{3}{4}\right)$ & 0 & 192 & -64 & 192 & 1152 & -192 & -64 & -192 & - & Dirichlet & $2,5,6,7$ \\
\hline$\left(\frac{5}{4}, \frac{5}{4}\right)$ & $-128 / 3$ & $320 / 3$ & $-320 / 3$ & $320 / 3$ & 0 & $1600 / 3$ & $-320 / 3$ & $1600 / 3$ & - & Dirichlet & $2,5,6,7$ \\
\hline$\left(\frac{3}{4}, \frac{5}{4}\right)$ & $64 / 3$ & -128 & 64 & 0 & 960 & -320 & $320 / 3$ & 320 & - & Dirichlet & $2,5,6,7$ \\
\hline
\end{tabular}

Table 4: Values of the weighting factors as a function of the interpolation location (Fig. 25 and the existent boundary condition.

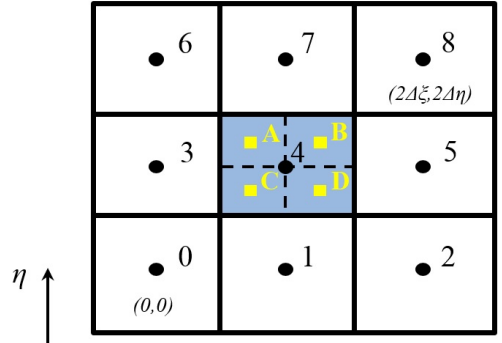

(a)

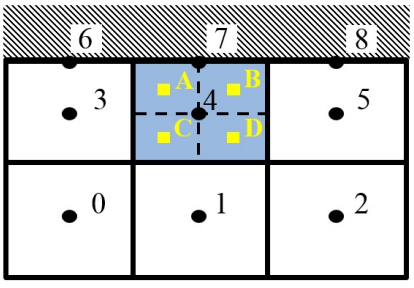

(b)

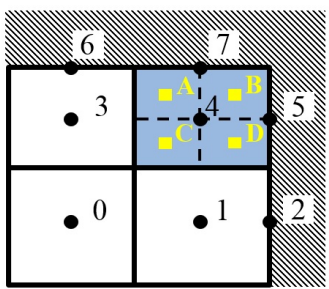

(c)

Fig. 25: Reconstruction stencils utilized to calculate values at the hanging nodes A, B , C and D within a reference cell. (a) No walls around the cell, (b) cell adjacent to one wall and (c) corner cell. • Cell centers; $\square$ hanging nodes 


\section{References}

[1] Anagnostopoulos, J. S., 2003. Discretization of transport equations on 2D Cartesian unstructured grids using data from remote cells for the convection terms. International Journal for Numerical Methods in Fluids 42 (3), 297-321.

[2] Angelidis, D., Assimakopoulos, V. D., Bergeles, G., 2015. A cartesian grid refinement method for simulating thermally stratified urban environments. Journal of Wind Engineering and Industrial Aerodynamics 142, 149 - 163.

[3] Angelidis, D., Sotiropoulos, F., 2015. Simulation of wind turbine wakes on locally refined cartesian grids. American Institute of Aeronautics and Astronautics, AIAA SciTech, 33rd Wind Energy Symposium.

[4] Anupindi, K., Delorme, Y., Shetty, D. A., Frankel, S. H., 2013. A novel multiblock immersed boundary method for large eddy simulation of complex arterial hemodynamics. Journal of Computational Physics 254, $200-218$.

[5] Balay, S., Abhyankar, S., Adams, M. F., Brown, J., Brune, P., Buschelman, K., Eijkhout, V., Gropp, W. D., Kaushik, D., Knepley, M. G., McInnes, L. C., Rupp, K., Smith, B. F., Zhang, H., 2014. PETSc Web page.

[6] Balay, S., Gropp, W. D., McInnes, L. C., Smith, B. F., 1997. Efficient management of parallelism in object oriented numerical software libraries. In: Arge, E., Bruaset, A. M., Langtangen, H. P. (Eds.), Modern Software Tools in Scientific Computing. Birkhäuser Press, pp. 163-202.

[7] Basumatary, M., Natarajan, G., Mishra, S. C., 2014. Defect correction based velocity reconstruction for physically consistent simulations of nonnewtonian flows on unstructured grids. Journal of Computational Physics 272, $227-244$.

[8] Benek J., Steger, J., Dougherty, F., 1983. A flexible grid embedding technique with application to the euler equations. American Institute of Aeronautics and Astronautics, AIAA 6th Computational Fluid Dynamics Conference Danvers.

[9] Benkenida, A., Bohbot, J., Jouhaud, J. C., 2002. Patched grid and adaptive mesh refinement strategies for the calculation of the transport of vortices. International Journal for Numerical Methods in Fluids 40 (7), 855-873.

[10] Berger, M., Colella, P., 1989. Local adaptive mesh refinement for shock hydrodynamics. Journal of Computational Physics 82 (1), 64-84.

[11] Berger, M. J., Oliger, J., 1984. Adaptive mesh refinement for hyperbolic partial differential equations. Journal of Computational Physics 53 (3), 484-512.

[12] Borazjani, I., Ge, L., Sotiropoulos, F., 2008. Curvilinear immersed boundary method for simulating fluid structure interaction with complex 3D rigid bodies. Journal of Computational Physics 227 (16), 7587 - 7620.

[13] Burton, T. M., Eaton, J. K., 2002. Analysis of a fractional-step method on overset grids. Journal of Computational Physics 177 (2), 336 - 364.

[14] Caruso, S., Ferziger, J., Oliger, J., 1986. Adaptive grid techniques for elliptic fluid-flow problems. AIAA-86-0498.

[15] Chamorro, L., Hill, C., Morton, S., Ellis, C., Arndt, R., Sotiropoulos, F., 2013. On the interaction between a turbulent open channel flow and an axial-flow turbine. Journal of Fluid Mechanics 716, 658-670.

[16] Chamorro, L., Port-Agel, F., 2009. A wind-tunnel investigation of wind-turbine wakes: Boundary-layer turbulence effects. Boundary-Layer Meteorology $132(1), 129-149$.

[17] Chen, W., Lien, F., Leschziner, M., 1997. Local mesh refinement within a multi-block structured-grid scheme for general flows. Computer Methods in Applied Mechanics and Engineering 144 (34), 327-369.

[18] Christlieb, A. J., Liu, Y., Tang, Q., Xu, Z., 2015. High order parametrized maximum-principle-preserving and positivity-preserving WENO schemes on unstructured meshes. Journal of Computational Physics 281, $334-351$.

[19] Coelho, P., Pereira, J. C. F., Carvalho, M. G., 1991. Calculation of laminar recirculating flows using a local non-staggered grid refinement system. International Journal for Numerical Methods in Fluids 12 (6), 535-557.

[20] Darbandi, M., Naderi, A., 2006. Multiblock hybrid grid finite volume method to solve flow in irregular geometries. Computer Methods in Applied Mechanics and Engineering 196 (13), $321-336$.

[21] de Nicola, C., Pinto, G., Tognaccini, R., 1995. On the numerical stability of block structured algorithms with applications to 1-d advection-diffusion problems. Computers Fluids 24 (1), $41-54$.

[22] de Zlicourt, D., Ge, L., Wang, C., Sotiropoulos, F., Gilmanov, A., Yoganathan, A., 2009. Flow simulations in arbitrarily complex cardiovascular anatomies an unstructured cartesian grid approach. Computers Fluids 38 (9), $1749-1762$.

[23] English, R. E., Qiu, L., Yu, Y., Fedkiw, R., 2013. An adaptive discretization of incompressible flow using a multitude of moving cartesian grids Journal of Computational Physics 254, 107-154.

[24] Fornberg, B., 5 1988. Steady viscous flow past a sphere at high reynolds numbers. Journal of Fluid Mechanics 190, $471-489$.

[25] Ge, L., Sotiropoulos, F., 2007. A numerical method for solving the 3D unsteady incompressible navier-stokes equations in curvilinear domains with complex immersed boundaries. Journal of Computational Physics 225 (2), 1782-1809.

[26] Gilmanov, A., Sotiropoulos, F., 2005. A hybrid cartesian/immersed boundary method for simulating flows with 3D, geometrically complex, moving bodies. Journal of Computational Physics 207 (2), 457 - 492.

[27] Gilmanov, A., Sotiropoulos, F., Balaras, E., 2003. A general reconstruction algorithm for simulating flows with complex 3D immersed boundaries on cartersian grids. Journal of Computational Physics 191 (2), 660-669.

[28] Hessenius, K. A., 1987. Conservative zonal schemes for patched grids in two and three dimensions. National Aeronautics and Space Administration, Ames Research Center.

[29] Hinatsu, M., Ferziger, J. H., 1991. Numerical computation of unsteady incompressible flow in complex geometry using a composite multigrid technique. International Journal for Numerical Methods in Fluids 13 (8), 971-997.

[30] Jeong, J., Hussain, F., 2 1995. On the identification of a vortex. Journal of Fluid Mechanics 285, 69-94.

[31] Johnson, T. A., Patel, V. C., 1999. Flow past a sphere up to a Reynolds number of 300. Journal of Fluid Mechanics 378, 19-70.

[32] Kang, S., Lightbody, A., Hill, C., Sotiropoulos, F., 2011. High-resolution numerical simulation of turbulence in natural waterways. Advances in Water Resources 34 (1), $98-113$.

[33] Kang, S., Yang, X., Sotiropoulos, F., 2014. On the onset of wake meandering for an axial flow turbine in a turbulent open channel flow. Journal of Fluid Mechanics 744, 376-403.

[34] Kazakidi, A., Tsakiris, D. P., Angelidis, D., Sotiropoulos, F., Ekaterinaris, J. A., 2015. CFD study of aquatic thrust generation by an octopus-like arm under intense prescribed deformations. Computers Fluids 115, $54-65$.

[35] Le Clair, B. P., Hamielec, A. E., Pruppacher, H. R., Mar. 1970. A Numerical Study of the Drag on a Sphere at Low and Intermediate Reynolds Numbers. Journal of Atmospheric Sciences 27.

[36] Lu, H., Port-Agel, F., 2011. Large-eddy simulation of a very large wind farm in a stable atmospheric boundary layer. Physics of Fluids 23 (6).

[37] MacNeice, P., Olson, K. M., Mobarry, C., de Fainchtein, R., Packer, C., 2000. PARAMESH: A parallel adaptive mesh refinement community toolkit. Computer Physics Communications 126 (3), 330-354.

[38] Magarvey, R. H., Bishop, R. L., 1961. Transition ranges for three-dimensional wakes. Canadian Journal of Physics 39 (10), $1418-1422$.

[39] Magarvey, R. H., MacLatchy, C. S., 1965. Vortices in sphere wakes. Canadian Journal of Physics 43 (9), 1649-1656.

[40] Magnaudet, J., Rivero, M., Fabre, J., 1995. Accelerated flows past a rigid sphere or a spherical bubble. Part 1. Steady straining flow. Journal of Fluid Mechanics 284, 97-135.

[41] Meakin, R., Suhs, N., 1989. Unsteady aerodynamic simulation of multiple bodies in relative motion. American Institute of Aeronautics and Astronautics, AIAA 9th Computational Fluid Dynamics Conference.

[42] Mittal, S., Jan. 2001. Computation of three-dimensional flows past circular cylinder of low aspect ratio. Physics of Fluids $13,177-191$.

[43] Nakamura, I., Jan. 1976. Steady wake behind a sphere. Physics of Fluids 19, 5-8. 
[44] Natarajan, R., Acrivos, A., 1993. The instability of the steady flow past spheres and disks. Journal of Fluid Mechanics 254, 323-344.

[45] Nezis, A., Angelidis, D., Assimakopoulos, V., Bergeles, G., 2011. On the wind flow patterns under neutral and unstable conditions in an urban area. International Journal of Environment and Pollution 47 (1-4), 257-267.

[46] Nicola, C. D., Pinto, G., Tognaccini, R., 1997. Stability of two-dimensional model problems for multiblock structured fluid-dynamics calculations Computers Fluids 26 (1), $43-58$.

[47] Norberg, C., 1 1994. An experimental investigation of the flow around a circular cylinder: influence of aspect ratio. Journal of Fluid Mechanics 258, 287-316.

[48] Papadakis, G., Bergeles, G., 1999. A local grid refinement method for three-dimensional turbulent recirculating flows. International Journal for Numerical Methods in Fluids 31 (7), 1157-1172.

[49] Pascarelli, A., Piomelli, U., Candler, G. V., 2000. Multi-block large-eddy simulations of turbulent boundary layers. Journal of Computational Physics 157 (1), $256-279$.

[50] Ploumhans, P., Winckelmans, G., Salmon, J., Leonard, A., Warren, M., 2002. Vortex methods for direct numerical simulation of three-dimensional bluff body flows: Application to the sphere at $\mathrm{Re}=300,500$, and 1000. Journal of Computational Physics 178 (2), 427 - 463.

[51] Quinlan, D., 2000. AMR++: A design for parallel object-oriented adaptive mesh refinement. Structured Adaptive Mesh Refinement (SAMR) Grid Methods 117, 53-58.

[52] Rai, M. M., 1986. A conservative treatment of zonal boundaries for Euler equation calculations. Journal of Computational Physics 62 (2), 472 503.

[53] Rimon, Y., Cheng, S. I., 1969. Numerical solution of a uniform flow over a sphere at intermediate Reynolds numbers. Physics of Fluids 12, 949-959.

[54] Roos, F. W., Willmarth, W. W., Feb. 1971. Some experimental results on sphere and disk drag. AIAA Journal 9, $285-291$.

[55] Sakamoto, H., Haniu, H., 1990. A study on vortex shedding from spheres in a uniform flow. Journal of Fluids Engineering-transactions of The Asme $112,386-392$

[56] Scarano, F., Poelma, C., 2009. Three-dimensional vorticity patterns of cylinder wakes. Experiments in Fluids 47 (1), 69-83.

[57] Sheldahl, R., Sotiropoulos, F., Conzemius, R. J., Wachtler, J. N., Strong, M. B., SAND80-2114, Sandia National Laboratories, Albuquerque, New Mexico, March 1981. Aerodynamic characteristics of seven airfoil sections through 180 degrees angle of attack for use in aerodynamic analysis of vertical axis wind turbines.

[58] Smagorinsky, J., 1963. General Circulation Experiments with the Primitive Equations. Monthly Weather Review $91,99$.

[59] Sørensen, J. N., Mikkelsen, R. F., Henningson, D. S., Ivanell, S., Sarmast, S., Andersen, S. J., 2015. Simulation of wind turbine wakes using the actuator line technique. Philosophical Transactions of the Royal Society of London A: Mathematical, Physical and Engineering Sciences 373 (2035).

[60] Sotiropoulos, F., Abdallah, S., 1991. The discrete continuity equation in primitive variable solutions of incompressible flow. Journal of Computational Physics 95 (1), $212-227$.

[61] Sotiropoulos, F., Yang, X., 2014. Immersed boundary methods for simulating fluid-structure interaction. Progress in Aerospace Sciences 65, 1 - 21.

[62] Starius, G., 1977. Composite mesh difference methods for elliptic boundary value problems. Numerische Mathematik 28 (2), $243-258$.

[63] Starius, G., 1980. On composite mesh difference methods for hyperbolic differential equations. Numerische Mathematik 35 (3), $241-255$.

[64] Steger, J. L., Benek, J. A., 1987. On the use of composite grid schemes in computational aerodynamics. Computer Methods in Applied Mechanics and Engineering 64 (1), $301-320$.

[65] Taneda, S., 1956. Experimental Investigation of the Wake behind a Sphere at Low Reynolds Numbers. Journal of the Physical Society of Japan 11, 1104.

[66] Tang, H. S., Jones, S. C., Sotiropoulos, F., Nov. 2003. An overset-grid method for 3D unsteady incompressible flows. J. Comput. Phys. 191 (2), $567-600$.

[67] Theodorakakos, A., Bergeles, G., 2001. A telescopic local grid refinement technique for wind flow simulation over complex terrain. Wind Energy 4 (2), 77-98.

[68] Tomboulides, A. G., Orszag, S. A., 2000. Numerical investigation of transitional and weak turbulent flow past a sphere. Journal of Fluid Mechanics $416,45-73$.

[69] Vanella, M., Rabenold, P., Balaras, E., 2010. A direct-forcing embedded-boundary method with adaptive mesh refinement for fluid-structure interaction problems. Journal of Computational Physics 229 (18), 6427-6449.

[70] Wang, M., Moin, P., 2002. Dynamic wall modeling for large-eddy simulation of complex turbulent flows. Physics of Fluids 14 (7), $2043-2051$.

[71] Williamson, C. H. K., Sep. 1989. Oblique and parallel modes of vortex shedding in the wake of a circular cylinder at low Reynolds numbers. Journal of Fluid Mechanics 206, 579-627.

[72] Williamson, C. H. K., 1992. The natural and forced formation of spot-like vortex dislocations in the transition of a wake. Journal of Fluid Mechanics 243, 393-441.

[73] Williamson, C. H. K., 1996. Three-dimensional wake transition. Journal of Fluid Mechanics 328, $345-407$.

[74] Wissink, A. M., Hornung, R. D., Kohn, S. R., Smith, S. S., Elliott, N., 2001. Large scale parallel structured amr calculations using the SAMRAI framework. In: Proceedings of the 2001 ACM/IEEE Conference on Supercomputing. Supercomputing '01. ACM, New York, NY, USA, pp. 6-6.

[75] Wu, J.-S., Faeth, G., 1993. Sphere wakes in still surroundings at intermediate Reynolds numbers. AIAA journal 31 (8), $1448-1455$.

[76] Wu, Y.-T., Port-Agel, F., 2013. Simulation of turbulent flow inside and above wind farms: Model validation and layout effects. Boundary-Layer Meteorology 146 (2), 181-205.

[77] Yang, X., Sotiropoulos, F., Conzemius, R. J., Wachtler, J. N., Strong, M. B., 2014, DOI: 10.1002/we.1802. Large-eddy simulation of turbulent flow past wind turbines/farms: the virtual wind simulator (VWiS). Wind Energy.

[78] Yang, X., Zhang, X., Li, Z., He, G.-W., Nov. 2009. A smoothing technique for discrete delta functions with application to immersed boundary method in moving boundary simulations. Journal of Computational Physics 228, 7821-7836.

[79] Zang, Y., Street, R. L., 1995. A composite multigrid method for calculating unsteady incompressible flows in geometrically complex domains. International Journal for Numerical Methods in Fluids 20 (5), 341-361. 